Borden et al., Thalamic state controls timing and synchronization of S1 cortex, 2021

\title{
Thalamic state controls timing and synchronization of primary somatosensory cortical representations in the awake mouse
}

\footnotetext{
${ }^{1}$ Wallace H Coulter Department of Biomedical Engineering, Georgia Institute of Technology and
} Peter Y Borden ${ }^{1^{*}}$, Nathaniel C Wright ${ }^{1^{*}}$, Arthur E Morrissette ${ }^{2}$, Dieter Jaeger ${ }^{2}$, Bilal Haider ${ }^{1}$, Garrett B Stanley ${ }^{1}$

\begin{abstract}
Emory University, Atlanta, GA, USA
\end{abstract}
${ }^{2}$ Emory University, Department of Biology, Atlanta, Georgia, USA

\section{*Denotes co-first authorship}

Abbreviated Title: Thalamic state controls timing and synchronization of S1 cortex

With 41 text pages, 8 Figures

Correspondence: Garrett B Stanley

Coulter Department of Biomedical Engineering

Georgia Institute of Technology \& Emory University

313 Ferst Drive

Atlanta GA 30332-0535

USA

Phone: 404-385-5037

Fax: 404-385-5044

Email: garrett.stanley@bme.gatech.edu

Conflict of Interest: The authors declare no competing financial interests.

Acknowledgements: This work was supported by NIH National Institute of Neurological Disorders and Stroke Grant R01-NS048285 (GBS), R01-NS104928 (GBS), U01-NS094302 (GBS and DJ), and NIH National Institute of Neurological Disorders and Stroke Pre-doctoral NRSA NS098691 (PYB). 
bioRxiv preprint doi: https://doi.org/10.1101/2021.07.09.451656; this version posted July 10, 2021. The copyright holder for this preprint (which

was not certified by peer review) is the author/funder, who has granted bioRxiv a license to display the preprint in perpetuity. It is made available under aCC-BY-NC 4.0 International license.

Borden et al., Thalamic state controls timing and synchronization of S1 cortex, 2021

\section{Summary (150 words max, currently 150$)$}

34 The thalamus controls transmission of sensory signals from periphery to cortex, ultimately 35 shaping perception. Despite this significant role, dynamic thalamic gating and the

36 consequences for downstream cortical sensory representations have not been well

37 studied in the awake brain. We optogenetically modulated the ventro-posterior medial

38 thalamus in the vibrissa pathway of the awake mouse, and measured spiking activity in

39 the thalamus, and at the level of primary somatosensory cortex (S1) using extracellular

40 electrophysiology and genetically encoded voltage imaging. Thalamic hyperpolarization

41 significantly amplified thalamic sensory-evoked spiking through enhanced bursting, yet

42 surprisingly the $\mathrm{S} 1$ cortical response was not amplified, but instead timing precision was

43 significantly increased, spatial activation more focused, and there was an increased 44 synchronization of cortical inhibitory neurons. A thalamocortical network model implicates

45 the precise timing of feedforward thalamic spiking, and timing-sensitive engagement of 46 synaptic depression, presenting a highly sensitive, state-dependent timing-based gating 47 of sensory signaling to cortex. 
Borden et al., Thalamic state controls timing and synchronization of S1 cortex, 2021

\section{INTRODUCTION}

50 We explore the world through our sensory periphery, where sensors transduce the signals

51 that ultimately give us perception of the world. The mammalian sensory thalamus gates

52 information from the periphery to primary sensory cortices, controlling what signals do

53 and do not make their way to cortex, thus playing a very critical role in sensing. Far from

54 a static relay, the thalamus is under continuous influence by modulatory inputs from

55 brainstem and feedback mechanisms from cortex (Sherman and Guillery, 2002;

56 Sherman, 2005), most extensively explored in the context of sleep and epileptic seizures

57 (Huguenard and McCormick, 2007; Fogerson and Huguenard, 2016). The large majority

58 of detailed thalamic studies focusing on its potential role in sensory signaling have been

59 either in ex-vivo/slice preparations or in intact/in-vivo preparations under anesthesia,

60 where prominent thalamic properties such as synchronization of convergent

61 thalamocortical projections and tonic/burst gating have been shown to strongly boost

62 signaling under these conditions (Murray Sherman, 2001; Sherman, 2001; Swadlow and

63 Gusev, 2001; Swadlow, 2002; Lesica and Stanley, 2004; Lesica et al., 2006; Wang et al.,

64 2010; Stanley et al., 2012; Whitmire et al., 2016, 2021). Recent studies exploring the role

65 of thalamus in the awake, unanesthetized brain have confirmed the critical role of

66 thalamus in grossly modulating sensory cortex (Halassa et al., 2011; Poulet et al., 2012a;

67 Lewis et al., 2015; Reinhold et al., 2015; Yu et al., 2016). However, whether increases in

68 thalamic excitability act to boost cortical sensory-evoked responses in the awake brain as

69 predicted from ex-vivo and anesthetized studies is unknown.

71 In addition to direct afferent inputs that serve as the primary drive of thalamocortical activity, thalamic nuclei receive more subtle modulatory inputs from a range of sources,

73 including corticothalamic feedback (McCormick and von Krosigk, 1992; Crandall et al., 2015a), thalamic reticular nucleus (Halassa and Acsády, 2016), as well as diffuse inputs

75 from the reticular formation (Castro-Alamancos, 2002) and reticular activating system (Rodenkirch et al., 2019) and other neuromodulatory centers (for a review, see Varela, 2014). The coordinated and collective effects of this complex array of excitatory and inhibitory inputs sets the operating point, or state, for thalamic neurons, which can vary widely in different conditions. These modulations in baseline thalamic excitability have important implications for both spontaneous and sensory-evoked thalamic firing activity, which interact to ultimately shape thalamic drive of cortex. Even small changes in baseline membrane potential have been shown to have appreciable effects on spontaneous firing

83 of thalamic neurons (Béhuret et al., 2015), setting the overall tone of synaptic drive to

84 cortex. The use of pharmacology to directly modulate thalamic state (Godwin et al., 1996;

85 Hirata and Castro-Alamancos, 2010; Poulet et al., 2012b) or opto/microstimulation and

86 pharmacology to indirectly affect thalamic state through cortical (Olsen et al., 2012;

87 Mease et al., 2014; Crandall et al., 2015b; Li and Ebner, 2016; Kirchgessner et al., 2020)

88 and subcortical (Aguilar and Castro-Alamancos, 2005) inputs has further causally 
revealed the sensitivity of cortex to overall thalamic drive. Importantly, thalamic neurons also exhibit distinct tonic versus burst firing modes (Sherman, 2001), the switching of which is highly sensitive to baseline membrane potential, and the de-inactivation of Ttype calcium channels (Suzuki and Rogawski, 1989; Kim et al., 2015). Neurons in the thalamus contain T-type calcium channels that are normally inactivated, but become activated through prolonged hyperpolarization. Subsequent depolarizing inputs lead to "bursts" of action potentials. Most actively investigated in the context of sleep states and rhythmic discharge, the role of this mechanism in sensory signaling remains unclear. Because thalamocortical high frequency bursting events have been shown to have a significant impact on downstream cortical activation (Swadlow and Gusev, 2001), the state-dependent gating of thalamic signaling through the aggregate effects of all these

100 properties is hypothesized to serve a critical role in processing sensory information (Crick, 1984; Sherman and Guillery, 2002; Sherman, 2005); however, this has not been investigated extensively in the intact brain due to the lack of methodological approaches to precisely manipulate thalamic state properties. To date, no studies have utilized temporally precise, repeatable and reversible modulation to precisely measure the effects of thalamic state and burst/tonic gating in awake cortex at the level of population signals and single neuron firing.

Here, we directly determined how thalamic gating properties control sensory-evoked thalamic and cortical responses in the vibrissa pathway of the awake, head-fixed mouse. Instead of driving or silencing neural activity, optogenetic manipulation was used to modulate thalamic state while recording extracellular thalamic and cortical activity and acquiring widefield cortical voltage imaging, using the voltage indicator ArcLight (Jin et al., 2012; Borden et al., 2017). We found that baseline thalamic firing rate was surprisingly invariant to optogenetic thalamic hyperpolarization through a transition from tonic to burst firing activity, and the baseline activity in S1 cortex was correspondingly invariant to thalamic hyperpolarization, following a brief transient increase in firing activity. In response to sensory stimuli, the thalamic hyperpolarization significantly amplified the

118 thalamic evoked response through enhanced bursting; however, the magnitude of the 119 response in S1 cortex was not amplified, but instead slightly attenuated relative to 120 baseline, despite the amplification in thalamic drive. However, the sensory evoked 121 response was also significantly more brief and spatially focused, and accompanied by an

122 increase in the synchronization of the putative cortical Fast-spiking (FS) inhibitory 123 neurons. A thalamocortical network model replicated these findings, and implicated the 124 precise timing of feedforward thalamic spiking, and the corresponding timing-sensitive 125 engagement of thalamocortical synaptic depression, as a likely mechanism underlying 126 the experimental observations. Taken together, the results here point to timing rather than 127 response magnitude as a fundamental feature of the thalamocortical circuit, presenting a dynamic, state-dependent timing-based gating of sensory signaling to cortex. 
Borden et al., Thalamic state controls timing and synchronization of S1 cortex, 2021

\section{METHODS}

131 All procedures were approved by the Institutional Animal Care and Use Committee at the

132 Georgia Institute of Technology and were in agreement with guidelines established by the

133 National Institutes of Health.

AAV Delivery: At least 5 weeks prior to experimentation, 6 week old female C57BL/6J

136 (Jackson Laboratories) mice were injected with different viral constructs either in the 137 ventral posteromedial (VPm) thalamic region with AAV-5-CamKinasell-eNph3.0 (UNC 138 Viral Vector core) for optogenetic modulation, in the primary somatosensory (S1) cortex 139 with AAV-1-hsyn1-ArcLight (UPenn Viral Vector Core) for optical voltage imaging, or both.

140 For surgical procedures, mice were anesthetized using isoflurane (3-5\%). After the 141 mouse was fully anesthetized, small craniotomies were placed over the regions of interest 142 and were aligned using stereotaxic measurements (For VPm, 1.8mm lateral from midline 143 by $1.8 \mathrm{~mm}$ caudal from bregma). For cortical expression, either single or multiple injection 144 sites were used surrounding the barrel cortex (centered at $1.5 \mathrm{~mm}$ caudal from bregma 145 and $3 \mathrm{~mm}$ lateral from midline). The virus was loaded into a modified Hamilton syringe 146 (701-N) with a 35 micron borosilicate glass pipette tip or a Hamilton Neuros Syringe. 147 The syringe was initially lowered to the corresponding depth below the surface (for VPm: $1483 \mathrm{~mm}$ and For S1: $0.5 \mathrm{~mm}$ ) and the tissue was allowed to rest for 1 minute before injection. 149 Both sites received injections of $0.5-1 \mu \mathrm{l}$ of viral construct at a flow rate of $0.1 \mu \mathrm{l} / \mathrm{minute}$. 150 After injection, the pipette remained in place for an additional 5 minutes before slowly 151 being removed from the brain. The bore holes were filled with either bone wax or left to 152 close naturally. Throughout injection, mice were kept warm using a water heating system 153 to maintain body temperature. See Supplemental Figure S1 for histological validation of expression of halorhodopsin in thalamus and ArcLight in S1.

Awake Animal Preparation: At least four weeks after ArcLight and eNphR3.0 viral 157 injection, mice were anesthetized under isoflurane and were implanted with a head-plate. 158 Over the course of 5-14 days preceding the first imaging experiment, mice were routinely 159 handled to gain familiarity with the imaging system and immobilization device. During this 160 acclimatization period, mice were head fixed for increasingly longer periods of time, from 16115 minutes to 1.5 hours. During stimulation of the whisker, mice were prevented from 162 interacting with the whisker stimulator by obstructing the path from the paws to the 163 whisker. Mice were rewarded with sweetened milk (Nestle, Ltd.) throughout imaging. After 164 at least 5 days of handling and acclimating, mice became tolerant to immobilization in the 165 headplate restraint system. During passive stimulation of the whiskers, the mice often 166 actively moved their whiskers. Therefore, the whisker stimulator was placed relatively 167 close $(5 \mathrm{~mm})$ to the face to prevent the whisker from slipping out of the manipulator; 
bioRxiv preprint doi: https://doi.org/10.1101/2021.07.09.451656; this version posted July 10, 2021. The copyright holder for this preprint (which

was not certified by peer review) is the author/funder, who has granted bioRxiv a license to display the preprint in perpetuity. It is made available under aCC-BY-NC 4.0 International license.

Borden et al., Thalamic state controls timing and synchronization of S1 cortex, 2021

168 however, the amplitude of the deflection was adjusted to maintain a consistent angular velocity (1200 deg/s).

Whisker Stimulation: Whisker stimulation was similar to that utilized previously (Borden et al., 2017). Briefly, individual vibrissae of the mice were deflected by a high fidelity (1 $\mathrm{KHz}$ ) galvanometer system (Cambridge Technologies). A whisker stimulus was applied by positioning the custom designed galvanometer $5-10 \mathrm{~mm}$ from the face and delivering an exponential sawtooth (rise and fall time $=5 \mathrm{~ms}$ ). The waveform stimulus velocity was taken by averaging the time to peak velocity of the stimulus. The velocity was adjusted based on distance from the face.

Thalamic and Cortical Electrophysiology: For thalamic electrophysiology, a small craniotomy was made over the primary whisker sensitive thalamic ventral-posterior medial (VPm) region of the mouse, around the injection site (see above). First, the VPm was mapped with a $2 \mathrm{MOhm}$ tungsten electrode (FHC) which was slowly lowered until $2.5 \mathrm{~mm}$ below the cortical surface. The mouse VPm was identified using both stereotaxic measurements and depth as well as electrophysiological features (such as latency, peak response, whisker selectivity). A neuron was determined to be located in the VPm if the peri-stimulus time histogram (PSTH) contained a peak response 3ms -10ms after a 1200 degree/s (deg/s) punctate single whisker stimulation and did not have a latency shift by more than $20 \mathrm{~ms}$ after $1 \mathrm{~s}$ of a $10 \mathrm{~Hz}$ adapting stimulus (Wang et al., 2010). The principal whisker was first determined using a manual probe to isolate the whisker with the largest evoked response. If further isolation was needed, the principal whisker was determined by the largest $30 \mathrm{~ms}$ PSTH response of the surrounding three whiskers. Note that VPm single units were difficult to isolate in the awake animal, limiting our sample size, but the multi-unit VPm activity is also reflective of the same findings. For cortical recordings, initial mapping was conducted using cortical ArcLight voltage imaging or intrinsic imaging (see below). Once the target cortical column (barrel) was identified and confirmed, a 32channel silicon probe (NeuroNexus) or single tungsten electrode was inserted. For both thalamic and cortical electrophysiology, after the conclusion of the study either a small 7uA 10s lesion, or a fluorescent dye was placed near the recording location and confirmed using post-mortem histological validation. Neuronal signals were band-pass filtered $(500 \mathrm{~Hz}-5 \mathrm{KHz})$, digitized at $30 \mathrm{KHz} /$ channel and collected using a 96-channel dataacquisition system (Blackrock Microsystems, Salt Lake City, UT, USA). For single unit recordings, offline spike sorting was accomplished using Plexon Offline Spike Sorter v4 (Plexon, Inc). For multi-unit data, we measured threshold crossings from the continuously recorded thalamic or cortical activity. Thalamic multiunit activity was captured using a threshold criterion of 5 standard deviations over the entire recording (Yang et al., 2016). For cortical multiunit recordings, a manual threshold was set based on each experiment. Additional data analysis utilized custom scripts using Matlab (Mathworks, Inc). 
210 Awake Cortical Fluorescent ArcLight Imaging: ArcLight transfected mice were imaged

211 through the thinned or removed skull using a two camera system: a Scimedia Imaging 212 system to measure cortical ArcLight spatial activity, and a custom camera to measure 213 hemodynamic activity for subtraction. The cortex was imaged using a $184 \times 123$ pixel CCD 214 Camera, MiCam2 HR Camera (Scimedia, Ltd) to capture ArcLight, and a Basler Ace 215 (acA1920-155um) $480 \times 180$ pixel (4x4 binned) CMOS Camera to capture auto216 fluorescence, at $200 \mathrm{~Hz}$ with a tandem lens microscope. The entire cortical area was 217 illuminated at $465 \mathrm{~nm}$ with a $400 \mathrm{~mW} / \mathrm{cm} 2$ LED system (Scimedia, Ltd.) to excite the 218 ArcLight fluorophore and background auto-fluorescence. The excitation light was 219 projected onto the cortical surface using the first dichroic mirror (bandpass: $475 / 625 \mathrm{~nm}$, 220 Semrock, Inc.). Collected light was passed through a second dichroic mirror (Longpass 221 cutoff: $495 \mathrm{~nm}$, Semrock, Inc.) for collection of the ArcLight and auto-fluorescence signal. 222 The auto-fluorescence signal was filtered with a bandpass filter between the wavelengths 223 of 465/75 nm (Semrock, Inc). The ArcLight signal was filtered with a bandpass emission 224 filter between wavelengths of $52035 \mathrm{~nm}$ (Semrock, Inc.). The imaging system was focused approximately $300 \mu \mathrm{m}$ below the surface of the brain or cortical layer $2 / 3$.

Anesthetized Cortical Fluorescent ArcLight \& Intrinsic Imaging: ArcLight transfected mice were imaged through the thinned or removed skull using a Scimedia Imaging system to measure cortical spatiotemporal activity (leveraging a single camera setup). The cortex was imaged using a 184x123 pixel CCD Camera, MiCam2 HR Camera (Scimedia, Ltd) at $200 \mathrm{~Hz}$, and a tandem lens macroscope. The entire cortical area was illuminated at $465 \mathrm{~nm}$ with a $400 \mathrm{~mW} / \mathrm{cm}^{2}$ LED system (Scimedia, Ltd.) to excite the ArcLight fluorophore. The excitation light was further filtered (cutoff: $472-430 \mathrm{~nm}$ bandpass filter, Semrock, Inc.) and projected onto the cortical surface using a dichroic mirror (cutoff: 495 $\mathrm{nm}$, Semrock, Inc.). Collected light was filtered with a bandpass emission filter between wavelengths of 520-535 $\mathrm{nm}$ (Semrock, Inc.). The imaging system was focused approximately $300 \mu \mathrm{m}$ below the surface of the brain to target cortical layer $2 / 3$. For intrinsic imaging of the hemodynamic response, the cortical surface was illuminated by a $625 \mathrm{~nm}$ red LED (ThorLabs), and imaged with the same camera system as above, at a temporal resolution of $10 \mathrm{~Hz}$. During intrinsic imaging, no emission filters were used. In order to evoke a cortical intrinsic response, the whisker was repetitively stimulated at $10 \mathrm{~Hz}$ for 6 seconds.

Functional Fluorescent Mapping of Barrel Cortex: The mouse's whisker system was first mapped by imaging the rapid ArcLight response to a high velocity (1200 Deg/s) sensory stimulus separately applied to three different whiskers. The resulting whisker response averaged over 20 trials was determined to be associated with a principal whisker, and 249 barrel, if the evoked response was spatially limited to roughly a $0.2 \mathrm{~mm} \times 0.2 \mathrm{~mm}$ area 
Borden et al., Thalamic state controls timing and synchronization of S1 cortex, 2021

251 from the barrel field if the center of mass of activation moved consistently with the 252 histologically defined barrel field and was within the standard stereotaxic location of S1 253 ( $\sim 3 \mathrm{~mm}$ lateral, $0.5-1.5 \mathrm{~mm}$ from bregma). After mapping, a single whisker was deflected 254 in a way as to emulate a high velocity slip-stick event (1200 deg/s), either with or without thalamic optogenetic hyperpolarization.

Simultaneous Imaging and Thalamic Optogenetic Manipulation: After mapping both the thalamic and cortical regions, an optrode (2M Ohm tungsten electrode mounted to an 200um optic fiber) was positioned to the stereotaxic locations of the pre-mapped thalamic region and lowered to the corresponding depth. Once a single thalamic unit was identified using the above constraints, the unit was determined to be sensitive to optical stimulation by briefly (1-2s) hyperpolarizing the cells using $\sim 17 \mathrm{~mW} / \mathrm{mm}^{2}$ (LED condition) or $\sim 35 \mathrm{~mW} / \mathrm{mm}^{2}$ (double LED condition) (unless otherwise noted) at $590 \mathrm{~nm}$ from an LED light source (Thorlabs, M590-F1). Each cell was determined to be a thalamic optically sensitive unit if the lighted caused a transient decrease in firing rate or if the cessation of the 590nm light caused a rebound burst (Brecht and Sakmann, 2002). After identifying an optically sensitive thalamic unit, the whisker stimulus was presented under various light conditions. Light stimulation was presented $500-750 \mathrm{~ms}$ preceding and following whisker deflection. There was at least a four second interval between stimulus deliveries to allow for recovery of halorhodopsin (eNphR3.0). Each session imaged 200ms-1s of frames preceding whisker stimulation to measure spontaneous activity. Prior to use, light power was measured from the tip of the ground optical fiber before each experiment to maintain approximate light intensities delivered to each cell. During light delivery, the downstream cortical response was recorded using either electrodes for cortical electrophysiology or voltage imaging as described above. The optogenetic and viral expression of each experiment was verified through confocal and brightfield imaging of fixed slices.

The LED light intensity used for optogenetic stimulation is within the published range of light stimulation $\left(35 \mathrm{~mW} / \mathrm{mm}^{2}\right.$ is estimated from our maximum power measure of $1.2 \mathrm{~mW}$ through a 200um fiber) (Stujenske et al., 2015; Owen et al., 2019). Further, studies that have directly quantified the effects of optical stimulation on local tissue heating and neural activation (in the absence of opsin expression) have found no significant difference in the firing rate change for $1 \mathrm{~mW}$ light intensity (Stujenske et al., 2015) or minor firing rate changes for $3 \mathrm{~mW}$ light intensity, but no behavioral effects (Owen et al., 2019). See Supplemental Figure S5 for controls that demonstrate a lack of light effects and lack of confounding interactions between optogenetic activation of VPm thalamus and GEVI imaging in cortical S1. light anesthesia, as a control. These mice were initially anesthetized using isoflurane (3- 
$2935 \%)$ and then placed on a heated platform ( $\mathrm{FHC}$, Inc.) in a stereotaxic nose cone to

294 maintain anesthesia. A large incision was placed over the animal's skull, and 295 the connective tissue and muscle surrounding the skull was removed using a 296 fine scalpel blade. A modified headplate was attached using dental acrylic (Metabond) 297 and secured to the skull. For cortical imaging, the skull was thinned with a dental drill until 298 transparent, or removed entirely and covered with saline or ringers solution. After surgery, 299 the isoflurane levels were dropped to $\sim<1 \%$ for imaging and electrophysiology, the 300 procedures for which were identical to those for the awake animal. The animal's vitals 301 (heart rate and respiratory rate) were constantly measured for tracking anesthesia depth.

Histology: Histological procedures were similar to those utilized previously (Borden et al., 2017), to validate ArcLight in S1 and/or opsin expression in VPm thalamus. Histological samples were prepared by perfusing the animal transcardially with phosphate buffered saline (PBS) followed by $4 \%$ paraformaldehyde. Brains were post-fixed overnight in $4 \%$ paraformaldehyde then transferred to PBS before sectioning. Thick sections were cut using a vibratome $(100 \mu \mathrm{m}$, Leica, VTS 1000) and either directly mounted or saved for staining. See Supplemental Figure $\mathrm{S} 1$ for histological validation of expression of halorhodopsin in VPm and ArcLight expression in cortex.

Thalamic Electrophysiology Data Analysis - Mean Response and Burst Ratio: We report several different basic measurements of spiking activity from our thalamic units including evoked response and evoked bursting response. Thalamic firing activity was reported as PSTHs with units of firing rate in $\mathrm{Hz}$, calculated as the number of spikes within a bin, divided by the size of the bin (see individual plots for bin size). We determined thalamic evoked response as the initial response $(0-30 \mathrm{~ms})$ to sensory stimuli, reported as the number of spikes per stimulus averaged over many trials (9-102 trials). The corresponding evoked bursting response was determined as number of bursting spikes per trial in that same post stimulus period. Bursting spikes were defined as 2 or more spikes that fire at most $4 \mathrm{~ms}$ apart preceded by $100 \mathrm{~ms}$ of silence. The $100 \mathrm{~ms}$ pre-stimulus activity is based on reported values for T-type calcium bursts (Swadlow and Gusev, 2001; Whitmire et al., 2016). All data analysis of the recorded extracellular thalamic units was accomplished using custom Matlab scripts.

Cortical Electrophysiology Data Analysis: Putative single cortical units were identified offline using open-source spike sorting software (KiloSort2). In order to be considered well isolated, each neural unit must have signal to noise ratio greater than 2 , and have less than $1 \%$ of spikes within $0-1 \mathrm{~ms}$ Inter-spike Interval (ISI). Although recordings originated across cortical layers, for simplicity, all units were analyzed in the same pool, regardless of the laminar origin. Individual single-units were classified as putative Regular Spiking Units (RSUs) or Fast Spiking Units (FSUs) based on the characteristics of the spike waveform (McCormick et al., 1985; Niell and Stryker, 2010; Guo et al., 2017; 
Speed et al., 2019; Yu et al., 2019). Specifically, units with a spike-width exceeding 0.4 $\mathrm{ms}$ (defined as trough-to-peak) were classified as RSUs, and below this as FSUs. Cortical firing activity was reported as PSTHs with units of firing rate in $\mathrm{Hz}$, calculated as the number of spikes within a bin, divided by the size of the bin (see individual plots for bin size). All spikes within a $1 \mathrm{~ms}$ ISI were removed from analysis. Synchrony across cortical single-units was computed from spike cross-correlograms across recorded pairs. Specifically, synchrony was defined as proportion of spikes from the full $(+/-100 \mathrm{~ms})$ cross-correlogram that were in a central $+/-7.5 \mathrm{~ms}$ window (Wang et al., 2010; Whitmire et al., 2016). To determine the number of needed synchronous events to accurately measure synchrony, we simulated two neurons with a $\sim 5 \%$ change in synchrony (assuming a normal distribution). We found that approximately 50 events were required to accurately separate the two distributions.

Voltage Imaging Data Processing: Raw images were loaded and converted from the SciMedia ". gsd" format using custom scripts and down-sampled by a factor of two. Each dataset was first normalized to a $\% \Delta \mathrm{F} / \mathrm{F}_{\mathrm{o}}$ measurement by subtracting and dividing each trial by the temporal average of the frames 0 to $200 \mathrm{~ms}$ preceding light delivery $\left(F_{0}\right)$. In two instances, where $200 \mathrm{~ms}$ of preceding LED onset activity was not captured, the Fo was taken as an average $200 \mathrm{~ms}$ period across no-stimulus trials. Hemodynamic noise was removed using a PCA background subtraction method. As described in detail (Borden et al., 2017), in vivo ArcLight imaging overlaps with the hemoglobin absorption spectrum, and therefore contains hemodynamic noise that must be removed for analysis. Imaging the wildtype mouse cortical surface using the same blue excitation and ArcLight filter set revealed similar patterns of oscillatory activity, likely through auto-fluorescence and effects of hemodynamic absorption and blood flow (Ma et al., 2016). The background PCA subtraction utilizes the auto-fluorescence signal from non-ArcLight transfected regions to predict the hemodynamic signal across the recorded space. Specifically, the method uses principal component analysis of non-expressing low background autofluorescence regions (determined from the maximum fluorescence from a non-injected animal) to find the ongoing hemodynamic components on a single trial basis. Additionally, the background fluorescence regions were selected at least $1 \mathrm{~mm}$ away from the recorded whisker evoked response (Borden at el, 2017). Ideally, these criteria would create a spatially defined region with little or no ArcLight fluorescence to isolate the hemodynamic signal from the signal of interest. Each frame is first spatially averaged by either a 200 $\mu \mathrm{m} \times 200 \mu \mathrm{m}$ circular averaging (pillbox) filter or a media filter to reduce noise. On a single trial, the corresponding top five principal components of the low background regions (which contains approximately $85 \%$ of the variance explained) are projected on a pixel by pixel basis across the entire recording using a lasso regression method with regularization. The lasso regression utilizes a cross-validated approach to determine the 
375 to prevent the removal of any stimulus evoked activity, each pixel was fit on pre-stimulus 376 activity (either before light onset for experiments involving optogenetics, or immediately 377 preceding stimulus delivery). The final predicted hemodynamic signal for each pixel was 378 subtracted across the entire recording on a pixel by pixel basis. Due to the complex waveform of the hemodynamic response, a simple notch filter is not effective at separating the signal from the noise (Borden et al., 2017). We found that the background PCA subtraction method greatly reduced hemodynamic signal across the entire frame, compared to the off-ROI method (Borden et al, 2017). In some instances, brief onset and offset light artifacts of the 590nm light was visible in the recorded ArcLight cortical signal. To account for any optogenetic transient light artifacts, we only considered the relative changes in fluorescence during steady state light levels. Both raw and processed images showed qualitatively similar results.

Awake Voltage Imaging Data Analysis -Dual Camera: In the awake animal, we utilized a dual camera imaging system to capture a background fluorescence signal for hemodynamic subtraction. Two different cameras were used to capture the ArcLight and auto-fluorescence signals, and therefore, pixels could not be directly registered for subtraction for pixel by pixel correction. Instead, we utilized the same Background PCA subtraction method to find and develop models of the hemodynamic response based on the global PCA signal derived from the background image. For the dual camera data, each component was fit over the entire recording for subtraction of the hemodynamic noise. Both raw and processed images showed qualitatively similar results. Unless otherwise noted, each dataset was processed with the Background PCA or Dual Camera subtraction method as stated above. We measured the effect of the optogenetic stimulation on the peak amplitude of the evoked mean ArcLight fluorescence in the determined cortical barrel. The cortical barrel region of interest $(\mathrm{ROI})$ for each stimulated barrel and each data set was selected as the $200 \mu \mathrm{m} \times 200 \mu \mathrm{m}$ region with the largest response 30ms after stimulus delivery. This determined ROI was used for all subsequent analyses of the temporal response. To better isolate the evoked amplitude, the frame preceding stimulus delivery ( $t=-5 \mathrm{~ms}$ ) was subtracted from the resulting recorded signal. For each recording, the peak amplitude was defined as the $\Delta F / F_{\circ}$ at the time of the maximum average response between 0 and $110 \mathrm{~ms}$ for the strongest stimuli $(1200 \mathrm{Deg} / \mathrm{s})$ presented under control and various optogenetic conditions. In order to measure the temporal properties of the evoked response, we concentrated on the timeseries data from the determined cortical barrel $\mathrm{ROI}$. For normalized fluorescence (Norm $\Delta \mathrm{F} / \mathrm{Fo}$ ), each session's peak response was divided by the average peak response to the strongest stimulus (1200 Deg/s) under the control condition. The normalization allows for a better comparison across animals which may have different levels of ArcLight expression. Peak time was defined as the time of 
416 the maximum fluorescence between 0 and $110 \mathrm{~ms}$ post stimulus, and the time of return 417 to baseline as the time when the fluorescence crossed the pre-stimulus baseline value 418 following the peak. Peak-to-baseline was then calculated as the time between the 419 fluorescence peak and the return of the fluorescence to baseline. Recovery was defined as the average fluorescence in the $120-400 \mathrm{~ms}$ window following the stimulus.

Imaging Data Analysis - Area Measurements: In addition to measuring the peak response, we also measured the effect of different thalamic states on the evoked area of sensory cortical activity. We measured the activated area as the number of pixels exceeding a threshold using the average response at the peak frame $(0-110 \mathrm{~ms})$ preceding stimulus delivery. Similar to other studies (Lustig et al., 2013; Millard et al., 2015), we measured the spatial activation using the $70 \%$ threshold. To compare the area independent of amplitude changes, we normalized the peak frame by dividing by the peak fluorescence in each condition (Control and LED). In order to isolate the evoked activity from ongoing activity, we subtracted the frame preceding stimulus delivery ( $t=-5 \mathrm{~ms}$ ). Different thresholds had no effect on the observed trends.

Whisking Recordings and Analysis: In all analyses, we included electrophysiological and voltage imaging data regardless of the behavioral state of the animal, which invariably combines data during whisking and non-whisking periods. As a control, we measured the awake whisking behavior using a Basler Ace (acA1920-155um) camera sampled with 480 $x 300$ pixel $(4 \times 4$ binned) at $20-50 \mathrm{~Hz}$. The whiskers were illuminated using either an external LED light $(860 \mathrm{~nm})$ or by the imaging $465 \mathrm{~nm}$ light source. Images were continually recorded through the entire recorded session and then aligned using the movement of the galvo stimulus. A select region of interest (ROI) was placed in the whisker pad close to the face to determine changes in average intensity during the recording session. A custom algorithm measured the squared change in intensity to determine periods of whisker movement. Whisker movement was assigned using a threshold of pixel intensity that was used for each imaging session. A movements had to last longer than $100 \mathrm{~ms}$ to considered as whisking periods. Any trial with a whisking periods within $500 \mathrm{~ms}$ of the stimulus were determined to be a whisking trial. As shown in Supplemental Figure S4, analyses performed on data parsed based on whisking confirm that the observed trends were consistent across whisking and non-whisking periods. derived from previously published models of thalamic function from the LGN (Smith et al., 2000; Lesica and Stanley, 2004; Lesica et al., 2006) and VPm (Whitmire et al., 2016). In order to simulate the experimental parameters and account for changes in thalamic activity, some additional terms and parameters were added and adjusted. Additionally, we generated ongoing activity using two methods, either injected current noise or synaptic 
Borden et al., Thalamic state controls timing and synchronization of S1 cortex, 2021

events, with both showing the same results. The results shown here use the synaptic event model where IPSCs and EPSCs are modulated as fixed inputs. The model itself was written and analyzed using custom scripts in Matlab 2016a.

The model is based on modifications of the standard integrate and fire model representing the effects of integrated synaptic currents on membrane voltage:

$$
\begin{gathered}
C \frac{d V}{d t}=I_{E P S C}-I_{I P S C}-I_{L}-I_{T}-I_{\text {Halo }} \\
I_{L}=g_{L}\left(V-V_{L}\right), I_{T}=g_{T} m_{\infty} h\left(V-V_{T}\right)
\end{gathered}
$$

The following parameters were used to simulate thalamic activity: $C=2 \mathrm{uF} / \mathrm{cm}^{2}, g_{L}=$ $0.035 \mathrm{mS} / \mathrm{cm}^{2}, g_{T}=0.07 \mathrm{mS} / \mathrm{cm}^{2}, \mathrm{~V}_{L}=-65 \mathrm{mV}, \mathrm{V}_{\text {reset }}=-45 \mathrm{mV}, \mathrm{V}_{h}=-68 \mathrm{mV}, \mathrm{V}_{T}=$ $120 \mathrm{mV}, \tau_{h}^{+}=0.1 \mathrm{~s}, \tau_{h}^{-}=0.02 \mathrm{~s}, \tau_{s}=1 \mathrm{e}-2 \mathrm{uF} / \mathrm{cm}^{2}$, Threshold $=-35 \mathrm{mV}, I_{\text {Halo }}=0-$ $1 u \mathrm{~A} / \mathrm{cm}^{2}$. EPSPs occurred at a rate $\mathrm{EPSP}_{\text {rate }}=1-25 \mathrm{~Hz}$, where each excitatory postsynaptic event had a peak of $3 \mathrm{nA} / \mathrm{cm}^{2}$, and decayed according to the above first-order differential equation for $\mathrm{I}_{E P S C}$. Similarly, IPSPS occurred at a rate of IPSP $_{\text {rate }}=0-5 \mathrm{~Hz}$, where each inhibitory post-synaptic event had a peak of $1 \mathrm{uA} / \mathrm{cm}^{2}$, and decayed according to the above first-order differential equation for $\mathrm{I}_{I P S C}$. To simulate the different levels of thalamic activity, we varied the rates of EPSP inputs on the thalamic model (based on published ranges of thalamic activity (Urbain et al., 2015)). IPSPs were simulated at a much lower rate ( $20 \%$ of EPSP rate) to add additional variability to baseline activity. The model outputs represent the average response of a 100 simulated thalamic neurons in response to various levels of thalamic hyperpolarization and baseline activity. The model was updated at $1 \mathrm{~ms}$ steps. The absolute refractory period was set to $1 \mathrm{~ms}$.

Cortical E-I Modeling: We constructed a simple model of the thalamocortical network using custom scripts written in Python 3.6.10, as described previously (Wright et al., 2021) 488 We modeled a single cortical barrel as a clustered network of excitatory and inhibitory 489 single-compartment leaky integrate-and-fire (LIF) neurons, subject to excitatory thalamic 490 and non-thalamic synaptic inputs. For both the "Control" and "LED" conditions, we 491 simulated 50 trials, each lasting $200 \mathrm{~ms}$, with a time-step of $0.05 \mathrm{~ms}$. A detailed 
bioRxiv preprint doi: https://doi.org/10.1101/2021.07.09.451656; this version posted July 10, 2021. The copyright holder for this preprint (which

was not certified by peer review) is the author/funder, who has granted bioRxiv a license to display the preprint in perpetuity. It is made available under aCC-BY-NC 4.0 International license.

Borden et al., Thalamic state controls timing and synchronization of S1 cortex, 2021

492 description of the model can be found in Cortical E-I Modeling Supplemental, Section 1.

493 All code is freely available upon request.

494

Non-zero thalamocortical (TC) synaptic weights were broadly distributed, and we implemented differential TC connectivity by imposing higher TC convergence (Bruno and Simons, 2002; Cruikshank et al., 2007) and shorter synaptic latencies (Cruikshank et al., 2007a; Kimura et al., 2010a) for inhibitory neurons, and requiring that VPm neurons with the highest rates synapsed only onto inhibitory neurons (Bruno and Simons, 2002).

We modeled a single cortical column as a network of 800 excitatory and 100 inhibitory LIF neurons, with relatively strong inhibitory-to-excitatory synapses (Gabernet et al., 2005). We imposed spatial clustering via "small-world" network connectivity (LitwinKumar and Doiron, 2012; Bujan et al., 2015; Wright et al., 2017a, 2017b), with 10\% rewiring probability. Inhibitory LIF neurons had shorter membrane time constants (Gentet et al., 2010) and refractory periods than excitatory neurons, which - together with the TC connection properties described above - supported higher firing rates in inhibitory neurons, as observed here (Fig. 6B, C) and in previous work (Bruno and Simons, 2002; Khatri et al., 2004; Gentet et al., 2010; Taub et al., 2013). Excitatory neurons were subject to an inhibitory spike-rate adaptation conductance, which helped to stabilize network 511 activity.

We drew tonic and burst thalamic spike times from the empirical VPm spike time PSTHs, with bursts modeled as either pairs (in the Control condition) or triplets (in the LED condition) of spikes with $2.5 \mathrm{~ms}$ ISI. In response to a spike in a given thalamic neuron, all TC synapses from that neuron decayed instantly and recovered slowly.

We employed four alternate models to parse the roles played by TC synaptic adaptation, changes in thalamic firing (i.e., burst ratio and the overall time-course of evoked firing), and intracortical inhibition. For each model, we calculated the grand mean $+/$ - SEM firing rates for all neurons, for a $50 \mathrm{~ms}$ pre-stimulus window, and an "early" $(0-50 \mathrm{~ms}$ poststimulus) and "late" (60 - 100 ms post-stimulus) response window, where stimulus onset time represents the time of galvo deflection onset, or $t=0$ in the empirical VPm PSTH.

524 For synchrony analysis, we randomly selected 200 excitatory and 200 inhibitory neuron 525 pairs.

Statistical Analysis \& Data Sharing: All tests were conducted using the MATLAB Statistics Toolbox (Mathworks, Inc.). For all measurements, we determined if the specific data sets were normally distributed using the Lilliefors test for normality. If the data were normal, we used the appropriate (paired or unpaired) t-test for statistical difference. If the population was determined to have non-normal distributions, we conducted non- 
parametric Wilcoxon signed-rank tests to determine statistical significance. All sample sizes are reported in the figure captions and Results text, along with an indication of particular test and the corresponding statistical significance level $\left({ }^{*}-p<0.05,{ }^{* *}-p<0.01\right.$, $* * *-p<0.001$ in figures). All datasets and software are available upon request.

\section{RESULTS}

All experiments were conducted in the vibrissa pathway of the mouse (Figure 1A). To directly test the predictions of previous in-vitro and anesthetized work, we conducted the first experiment in the isoflurane-anesthetized mouse (illustrated in Figure 1B). In the lightly anesthetized mouse, we utilized extracellular electrodes to record whisker-evoked spiking activity in VPm thalamus, in the presence (LED, $\sim 17 \mathrm{~mW} / \mathrm{mm}^{2}, 590 \mathrm{~nm}$ ) and absence (Control) of a hyperpolarizing optogenetic modulation of excitatory thalamic relay neurons expressing halorhodopsin (eNphR3.0, see Supplemental Figure S1), through a small fiber optic cable attached to the electrode (Figure 1B). Note that the inhibitory opsin was engaged with a constant illumination at a range of relatively low light levels, to induce sustained hyperpolarization, as opposed to complete inactivation. Note also that the total amount of light delivered here was in a range that has been previously shown to not induce significant heating of the surrounding tissue (see Methods), and control experiments indicate that there is no effect of the light alone in the absence of opsin expression (Supplemental Figure S5). Although the in-vivo optogenetic implementation precludes precise knowledge of the degree of hyperpolarization of the thalamic neurons due to variations in opsin expression, position of optic fiber relative to cells, etc., a separate set of in-vitro, slice experiments showed that VPm neurons were hyperpolarized by $\sim 15-25 \mathrm{mV}$ for the light levels utilized (see Supplemental Figure S2). Given that in the in-vitro experiments, light was presented more directly to the VPm neurons expressing halorhodopsin, and that in the in-vivo experiments the presentation of light did not completely silence the neurons, it is likely that the in-vitro experiments were an upperbound for the in-vivo case, and the actual hyperpolarization induced in-vivo was less than that of the in-vitro experiments.

Neurons in VPm thalamus responded to a single, computer-controlled punctate whisker stimulation (1200 deg/sec, sawtooth waveform) with a brief, transient sequence of action potentials (Figure 1D, see Methods for single unit and multi-unit classification). The sensory evoked thalamic VPm response was significantly amplified by optogenetic thalamic hyperpolarization, as shown in Figure 1D (all spikes in black, putative T-type calcium "burst" spiking in red, classified according to the criterion in Figure 1C). Shown in the insets are the low baseline firing rates in the anesthetized condition. Overall, there was a $\sim 35 \%$ increase in the evoked response from the Control to the LED condition 
572 (Figure 1E left, black, $p=0.0046$, paired Wilcoxon signed-rank, $n=29$ units), in large part 573 driven by the increase in sensory evoked thalamic bursting (Figure 1E left, red, $p=4.5 \mathrm{e}-$ 574 4, paired Wilcoxon signed-rank, $n=29$ units). In a subset of experiments, we recorded the 575 downstream cortical multiunit activity (Figure 1E right), and found a corresponding 576 increase during the period of hyperpolarization ( $\mathrm{n}=102$ trials, 2 recordings, 1 mouse). 577 Additionally, we simultaneously recorded cortical S1 activation at a meso-scopic scale 578 with optical voltage imaging in primary somatosensory (barrel) cortex using a Genetically 579 Engineered Voltage Indicator (GEVI, ArcLight, Borden et al., 2017) during optogenetic 580 hyperpolarization of thalamus (see Methods). This approach enables the characterization

581 of the overall spatiotemporal changes in S1 due to the thalamic manipulation 582 (Supplemental Figure S5). The imaging was focused at a cortical depth consistent with 583 layer $2 / 3$, where it captured sub-threshold activity reflective of suprathreshold layer 4 input 584 to layer 2/3 (Petersen et al., 2003). The fluorescence imaging of cortical S1 confirmed a 585 corresponding amplification of the sensory evoked response during thalamic 586 hyperpolarization (raw \%dF/Fo), as shown in an example session for the fluorescence 587 images in Figure $1 \mathrm{~F}$ and further quantified across the larger dataset in the left panel of 588 Figure 1G (normalized relative peak evoked response, $\sim 60 \%$ increase, $1 \mathrm{G}$ inset raw 589 relative peak evoked \%dF/Fo, $\mathrm{p}=0.017$, paired Wilcoxon signed-rank, $\mathrm{n}=13$ recordings 590 across 9 mice). There was an amplification in the peak response, followed by a relatively 591 fast transient decrease in fluorescence, followed by a gradual return to baseline over 592 approximately $200 \mathrm{~ms}$. The results here using optogenetic manipulation of thalamus and 593 wide-field voltage imaging of cortex are consistent with previous observations of 594 spontaneous thalamic bursting and the impact on downstream synaptic targets in cortical 595 layer 4 (Swadlow and Gusev, 2001). Importantly, the amplification in the peak response 596 observed here under these conditions reflects what would have been expected from the 597 observations in thalamus, if the cortical sensory representation were solely shaped by the 598 amplitude of the feedforward thalamic inputs.

600 Thalamic State and Ongoing Thalamic and Cortical activity in the awake brain

601 In a next set of experiments, we sought to characterize the influence of thalamic state 602 properties on gating of signaling to cortex in the awake mouse. Before turning to the 603 sensory-evoked responses, we first probed the effect thalamic hyperpolarization has on 604 baseline, ongoing activity that is significantly higher in the awake state as compared to 605 under anesthesia. The baseline firing rate in the awake state is significantly higher than 606 in the anesthetized condition, and we thus expect to see some effect of the optogenetic 607 hyperpolarization on baseline firing. An example single-unit VPm recording (experimental 608 setup in Figure 2A) is shown in the raster plot in Figure 2B. VPm neurons in awake mice 609 exhibited moderate ongoing firing activity $(4.9 \mathrm{~Hz}$ for this example). Upon presentation of 610 a ramping optical input to thalamus (LED, 590nm, $\sim 17 \mathrm{~mW} / \mathrm{mm}^{2}$ ), the spiking activity 611 underwent a qualitative change to a more sparse pattern of multiple action potentials, 
612 typical across all recorded cells. Bursts were present in the baseline (Control, no light) 613 condition, but substantially more prevalent in the presence of the hyperpolarizing 614 optogenetic input. This effect is summarized across all clearly identified single-units in the 615 top panel of Figure $2 \mathrm{C}(\mathrm{n}=5)$, showing the average instantaneous firing rate for all spiking 616 in black, and for the putative burst spiking in red (bands represent $+/-1$ standard error of 617 the mean, SEM). This shows that following an initial decrease in firing rate at the onset 618 of the light ramp, the VPm firing activity returned to the same overall pre-stimulus baseline 619 firing rate. This was accompanied by a monotonic increase in the burst firing rate, 620 suggesting that the return to pre-stimulus baseline firing rate was due to a compensatory 621 transition from tonic to burst firing. Note that the VPm nucleus in mice is a very small 622 structure, difficult to target and difficult to identify and maintain clearly isolated single units 623 in the awake state, thus we conservatively classified several of the recordings as multi624 unit in nature, which precludes burst/tonic identification. This same net effect we observed 625 in the single units is also shown in the bottom panel of Figure $2 \mathrm{C}$ for the multi-unit data 626 ( $n=9$ recordings). The multi-unit firing rate did not significantly change from the Control $627\left(0 \mathrm{~mW} / \mathrm{mm}^{2}\right)$ to the hyperpolarized condition (Figure 2D left, multi-unit, Control vs LED $p=$ 628 0.098, Control vs Double LED $\mathrm{p}=0.65$, Wilcoxon signed-rank, $\mathrm{n}=9 \mathrm{MU}$ recordings; $629 \sim 17 \mathrm{~mW} / \mathrm{mm}^{2}$ - LED or $\sim 35 \mathrm{~mW} / \mathrm{mm}^{2}$ - Double LED). Note that the firing rate in the LED 630 conditions was calculated from the $250-750 \mathrm{~ms}$ window, after the initial transient decrease 631 in firing rate. This lack of sustained effect on the overall thalamic firing rate was also 632 evident in the single-units (Figure 2D, middle, in the Control condition ( $n=8$ units) as 633 compared to the activation of the halorhodopsin ( $n=5$ units), for both all spikes (black) 634 and spikes classified as belonging to a burst (red). While there was no significant change 635 in the overall single-unit firing rate (black, Control vs LED $p=0.55$, Control vs Double LED $636 p=0.37$, unpaired t-test), there was a significant increase in the firing rate associated with 637 bursting (red, Control vs LED $p=0.014$, Control vs Double LED $p=0.032$, unpaired t-test), 638 indicating a compensatory shift from tonic to burst firing with the thalamic 639 hyperpolarization resulted in a significant increase in the burst ratio metric from the 640 Control to hyperpolarized condition (Fig. 2D, right, Control vs LED $p=0.0016$, Control vs 641 Double LED $p=0.0016$, unpaired Wilcoxon rank sum test). Further examination showed 642 that increasing hyperpolarization also increased the number of spikes per burst 643 (illustrated in left of Figure 2E), which partially offsets the loss of tonic spiking (right of 644 Figure 2E, Control vs LED $p=0.048$, Control vs Double LED $p=0.016$, unpaired Wilcoxon 645 rank sum test, $\mathrm{n}=5$ units across conditions, only considered units that had bursts).

Although it is not surprising that the thalamic burst mechanism was engaged by the 648 optogenetic hyperpolarization, it is surprising that the net baseline firing rate was not 649 decreased, but was instead invariant to the thalamic hyperpolarization. This effect was 650 not as apparent in the anesthetized condition when the baseline thalamic firing rate is 651 near zero, but emerges in the awake state where baseline thalamic firing rate is 
652 significantly higher. To investigate possible causes, we constructed an integrate-and-fire 653 or burst (IFB) model of thalamic firing that has been utilized in previous studies (Smith et 654 al., 2000; Lesica and Stanley, 2004; Lesica et al., 2006), where the resting membrane 655 potential was set by the level of activation of the hyperpolarizing opsin (see Methods). 656 When the model neuron was set to have a spontaneous, baseline firing rate consistent 657 with our observations $(\sim 5 \mathrm{~Hz})$, the effect of the hyperpolarizing opsin activation indeed 658 replicated this phenomenon (Figure 2F). An initial decrease in firing rate at the onset of 659 the light ramp was followed by a return to pre-light baseline level of firing (compare with 660 Figure 2C). Moreover, as in the experimental observation, the return to the baseline firing 661 rate in the hyperpolarized condition is dominated by the increase in bursting activity in the 662 IFB model (Figure 2F, red). The exact combination of the baseline activity and intensity 663 of the hyperpolarizing input strongly influenced the net resultant effect (Supplemental 664 Figure S3). Prolonged periods of halorhodopsin activation have been shown to potentially 665 alter the reversal potential of chloride (Raimondo et al., 2012). However, due to the short 666 timescales of LED stimulation used in this work (1-1.5s), and the large transition to burst 667 firing, the effect shown here is more likely driven by low voltage activation of T-type 668 calcium channels, further reinforced by the IFB model.

669

670 To uncover the downstream effects of the above observations in VPm, we performed the 671 same optogenetic manipulations during multi-electrode recording of single-unit activity in $672 \mathrm{~S} 1$ in the awake mouse (Figure 3A). Shown in the top panel of Figure 3B is a raster plot 673 from one example session (10 trials each from 15 simultaneously recorded units), in 674 response to the optogenetic hyperpolarization of thalamus with the LED (again 590nm, $67517 \mathrm{~mW} / \mathrm{mm}^{2}$ ). This example demonstrates a clear transient increase in spiking with the 676 thalamic hyperpolarization, typical of the cortical recordings. The bottom panel of Figure 677 3B shows the aggregate single-unit firing rate, with a clear transient increase in firing rate 678 at the light onset (bands represent $+/-1$ SEM), with a gradual return to the baseline, pre679 hyperpolarization level of firing rate at steady-state. Figure $3 \mathrm{C}$ summarizes this trend, 680 showing the mean single-unit firing rate across recorded cortical single-units in the 681 Control condition $\left(0 \mathrm{~mW} / \mathrm{mm}^{2}\right)$ versus activation of halorhodopsin in thalamus (LED $68217 \mathrm{~mW} / \mathrm{mm}^{2}$ or Double LED $-35 \mathrm{~mW} / \mathrm{mm}^{2}$ ), for the initial increase in firing rate during a 683 transient period (300-350 ms, open symbols) and the subsequent return to baseline firing 684 rate at steady state $(700-750 \mathrm{~ms}$, closed symbols). There was a significant transient 685 increase in the cortical firing rate across both led conditions (transient, Control vs LED $686 \mathrm{p}=5.4 \mathrm{e}-14$, Control vs Double LED $\mathrm{p}=1.4 \mathrm{e}-15$, Wilcoxon signed-rank test, $\mathrm{n}=118$ units). 687 After the transient increase, we observed a subsequent return to near baseline, either 688 with no net effect or a marginal increase in the rate (steady-state, Control vs LED $p=0.2$, 689 Control vs Double LED $p=0.013$, Wilcoxon signed-rank test). Interestingly, following 690 thalamic hyperpolarization, the cortical firing rate appeared to begin its initial transient 691 increase while the thalamic activity remained relatively low (Figure 2), which could reflect 
bioRxiv preprint doi: https://doi.org/10.1101/2021.07.09.451656; this version posted July 10, 2021. The copyright holder for this preprint (which

was not certified by peer review) is the author/funder, who has granted bioRxiv a license to display the preprint in perpetuity. It is made available under aCC-BY-NC 4.0 International license.

Borden et al., Thalamic state controls timing and synchronization of S1 cortex, 2021

692 an increased sensitivity of the thalamocortical synapse following the initial period of

693 quiescence (Swadlow and Gusev, 2001; Swadlow et al., 2005)

694

695

Taken together, the results here suggest that in response to the optogenetically induced thalamic hyperpolarization, the thalamic VPm neurons in the awake mouse exhibit a transient decrease in baseline firing rate, followed by a return to approximately prehyperpolarization level that is at least partially explained by a trade-off between tonic and burst firing, while the downstream activity in cortical S1 exhibits a transient increase in baseline firing rate, followed by an eventual return to approximately the original baseline

701 rates.

702

703

\section{Thalamic State and Sensory Evoked Thalamic and Cortical Activity}

704 We next recorded the sensory evoked single-unit activity in VPm thalamus in the presence and absence of thalamic hyperpolarization in the awake mouse (Fig. 4A). Neurons in VPm thalamus responded to a single, computer-controlled punctate whisker stimulation (1200 deg/sec, sawtooth waveform) with a brief, transient increase in multiunit activity (see Methods), which was significantly boosted with thalamic hyperpolarization (Fig. 4B; PSTH across multi-units, LED approximately $17 \mathrm{~mW} / \mathrm{mm}^{2}, \mathrm{n}=9$ recordings, 2 mice). Overall, there was an approximately $85 \%$ increase in the sensory evoked response of the thalamic MU VPm neurons with optogenetic hyperpolarization (Figure 4C, Control vs LED $p=0.0039$, Control vs Double LED $p=0.0039$, paired Wilcoxon signed-rank, $n=9$ recordings, 2 mice). To explore the potential role of tonic/burst gating in the boosting of the thalamic response, we analyzed clearly identified VPm single-units where spiking could be classified as tonic or burst in origin. The single-units also exhibited a brief, transient sequence of action potentials (Fig. 4D; PSTH across single-units, $n=8$ for Control, $n=5$ for LED approximately $17 \mathrm{~mW} / \mathrm{mm}^{2}$ ), qualitatively similar to the multi-unit data and to single-units observed in the anesthetized case. The top plot shows the peristimulus time histogram (PSTH, 2 ms bins) for all spikes (black) and putative burst spikes (red). In response to the transient sensory input, in the no-light (Control) condition, there is a short-latency transient VPm spiking response (black), with a modest level of putative T-type calcium bursting (red). In the presence of the hyperpolarizing light input to thalamus (Figure 4D, bottom, LED), however, there is an obvious increase in the VPm response to the sensory input (black), driven by a dramatic increase in bursting response (red), that is qualitatively similar to that in the anesthetized condition. The latency of the sensory-evoked response also increased, consistent with the T-type calcium channel bursts. We tested a range of LED intensities to determine the impact of various levels of thalamic hyperpolarization on the evoked bursting (Figure 4E; LED approximately $17 \mathrm{~mW} / \mathrm{mm}^{2}$, and Double LED approximately $35 \mathrm{~mW} / \mathrm{mm}^{2}$, for full range see Supplemental S6). With thalamic hyperpolarization, there was a significant increase in single-unit burst ratio (Figure 4E, Control vs LED $p=0.011$, Control vs Double LED $p=0.019$, unpaired 
Borden et al., Thalamic state controls timing and synchronization of S1 cortex, 2021

732 Wilcoxon rank sum, $\mathrm{n}=5$ units in 2 mice for (single \& double) LED conditions, $\mathrm{n}=8$ units in

7333 mice for Control). Taken together, the multi- and single-unit data reveal a boosting of

734 the sensory evoked thalamic response with thalamic hyperpolarization that is likely due

735 to the increased sensory-evoked thalamic bursting.

To examine the downstream consequences of the observed changes in the sensoryevoked response in thalamus, we recorded cortical S1 activation with wide-field optical voltage imaging during optogenetic hyperpolarization of thalamus in the awake mouse (illustrated in Figure 4F, see Methods). Note further that fluorescent imaging approaches like this are differential in nature (responses are based on relative not absolute changes in fluorescence), and are thus not well suited to capture overall absolute levels of ongoing background activity, but instead are targeting evoked responses relative to background. Based on previous findings (Swadlow and Gusev, 2001), the results in the anesthetized mouse, and the amplified sensory-evoked response in thalamus with thalamic hyperpolarization in the awake mouse, we expected to see a corresponding amplification of the cortical sensory-evoked response. Shown in Figure 4F is a trial averaged sequence of the sensory-evoked fluorescence images. Following the deflection at time 0 , a localized change in fluorescence began to emerge 10-15 ms later. Surprisingly, thalamic hyperpolarization did not result in an increased cortical response, but instead induced an apparent modest decrease in sensory evoked S1 activity. As quantified in Figure 4G, the peak fluorescence showed an approximately $20-40 \%$ decrease with this level of thalamic hyperpolarization (Control vs LED $p=0.020$, Control vs Double LED $p=0.0039$, paired Wilcoxon sign rank test, 2 mice over 9 sessions for LED conditions). Note that the right panel of Figure $4 G$ is the same data as in the left panel, with each dataset normalized to the Control condition, to show a percent decrease in the peak fluorescence.

Finally, in a separate set of experiments, we hyperpolarized thalamus while simultaneously recording the S1 Cortical SU responses using a multichannel electrode (illustrated in Figure 4H). The corresponding PSTHs for responses to a punctate whisker stimulus (1200 deg/sec, sawtooth waveform) are shown in Figure $4 \mathrm{I}$ (Control $\left(0 \mathrm{~mW} / \mathrm{mm}^{2}\right.$ ) vs. LED $\left(17 \mathrm{~mW} / \mathrm{mm}^{2}\right)$ conditions). The average absolute evoked cortical response remained approximately invariant during LED conditions across all cortical recorded units (Figure 4J, left, 5 to $100 \mathrm{~ms}$ post-stimulus, $\mathrm{n}=118$ ). However, the evoked cortical spiking relative to baseline decreased with increasing LED intensities (Control vs LED $p=0.20$, Control vs Double LED $p=0.034$, paired Wilcoxon signrank, $n=118$ units, across 3 mice), consistent with the GEVI recordings (Figure 4G), which are relative measures by construction.

769

To summarize, there is an amplification of the sensory evoked thalamic response with thalamic hyperpolarization, driven by the increase in sensory evoked bursting. Given this 
Borden et al., Thalamic state controls timing and synchronization of S1 cortex, 2021

772 amplification of the thalamic sensory evoked response that serves as the primary

773 feedforward inputs to cortical S1, it was surprising to observe in the awake mouse not an

774 increase, but instead a sensory evoked cortical response that was invariant in absolute

775 amplitude and decreased relative to background activity.

776

777

778

779

780 The GEVI imaging of cortex enables further investigation of the spatial characteristics of

781 the observed phenomenon. Figure 5A shows the imaging frame associated with the peak

782 sensory-evoked fluorescence, for the Control (top) and thalamic hyperpolarization (LED,

783 bottom) conditions. What is apparent qualitatively from the images is that there was an

784 overall attenuation of the cortical evoked response with thalamic hyperpolarization, as

785 previously shown in Figure 4. Less immediately obvious is the effect of the thalamic

786 hyperpolarization on the spatial extent of the sensory evoked cortical activation, as an

787 overall loss in amplitude would trivially produce an apparent loss in spatial activation, as

788 illustrated conceptually in the top panel of Figure 5B - often referred to as the "iceberg"

789 effect. The bottom panel of Figure 5B shows conceptually the alternative - that the

790 reduction in spatial area of activation may not just be due to the reduction in amplitude of

791 the evoked response, but instead can reflect a spatial sharpening of the sensory evoked

792 response with increasing thalamic hyperpolarization after accounting for the decrease in

793 peak amplitude of fluorescence. For the GEVI recordings here, the latter is indeed the

794 case - this is summarized as a reduction in the evoked normalized area of activation in

795 the left panel of Figure 5D (Control vs LED $p=0.025$, Control vs Double LED $p=0.046$,

796 paired t-test, 2 mice over 9 recording sessions for LED conditions).

797

798 In addition to the spatial characteristics of the sensory evoked response revealed by the

799 voltage imaging, the voltage imaging is also useful in revealing relatively fast temporal

800 dynamics of the aggregate cortical activity. Figure 5C shows the integrated fluorescence

801 signal within a barrel column in the presence (LED condition) or absence (Control) of light

802 input to activate halorhodopsin in thalamus, giving a picture of the temporal dynamics of

803 the sensory-evoked clear response to the punctate whisker stimulus (2 mice over 9 804 recording sessions). We again found a decrease of the sensory evoked response (note 805 that the fluorescence in Fig. $5 \mathrm{C}$ is normalized) despite a $\sim 85 \%$ increase in the sensory806 evoked multi-unit response in thalamus as measured in comparable experiments (Fig. 4). 807 Also apparent is the presence of a more rapid, post-peak decay in the fluorescence with 808 thalamic hyperpolarization, and a prolonged period of sub-baseline fluorescence before 809 returning to baseline after several hundred milliseconds. To quantify the temporal 810 sharpening of the sensory evoked response, we calculated the temporal width of the 811 evoked response as the time between the fluorescence peak and the first return to 
812 baseline level (defined as peak-to-baseline, Figure 5C), as shown in the middle panel of 813 Figure 5D, revealing a clear narrowing/sharpening of the temporal response as a function 814 of thalamic hyperpolarization from $\sim 150 \mathrm{~ms}$ in the Control condition, to $\sim 67 \mathrm{~ms}$ in the 815 thalamic hyperpolarized condition (Control vs LED $p=0.0059$, Control vs Double LED $816 \mathrm{p}=0.0019$, unpaired t-test, LED conditions $\mathrm{n}=9$, Control $\mathrm{n}=8$ recording sessions, note one 817 Control recording did not return to a pre-stimulus baseline). To quantify the prolonged 818 post-stimulus undershoot of activity, we calculated the overall fluorescence in a window 819 from 120 to $400 \mathrm{~ms}$ post-stimulus (defined as mean recovery in Figure 5C, Control vs LED $820 \mathrm{p}=0.016$, Control vs Double LED $\mathrm{p}=0.020$, paired $\mathrm{t}$-test, $\mathrm{n}=9$ recording sessions), as 821 shown in the right panel of Figure 5D. The increasingly negative relative fluorescence in 822 this window revealed a clear increase in the undershoot with increasing thalamic

823 hyperpolarization. Taken together, these S1 voltage imaging results show that in addition 824 to the attenuation of the sensory-evoked response with thalamic hyperpolarization, there 825 is a corresponding sharpening of the spatial activation of S1 and a temporal sharpening 826 in the form of a more transient evoked response with an inhibitory undershoot.

\section{Thalamic State and the Cortical Spike Timing}

829 Taken together, the results from Figure 5 revealed that the thalamic hyperpolarization 830 sharpens both the spatial and temporal aspects of the cortical sensory evoked response 831 in the awake brain. The absence of these effects in the anesthetized brain in Figure 1 832 suggests the potential role of cortical circuitry in shaping the sensory representations, as 833 isoflurane as an anesthetic has been shown to significantly suppress intracortical 834 dynamics (Greenberg et al., 2008; Vizuete et al., 2012; Haider et al., 2013; Aasebø et al., 835 2017; Suzuki and Larkum, 2020). To further probe this phenomenon, we parsed our 836 cortical single-units into putative excitatory neurons (regular spiking units, RSUs) and 837 putative inhibitory neurons (fast spiking units, FSUs). Specifically, single-unit activity was 838 identified (see Methods) and parsed based on RSU and FSU classification and combined 839 across cortical layers, the result of which is shown in Figure 6A. The top panel shows the 840 waveform for typical putative RSU (red) and FSU (blue), where the solid line represents 841 the mean waveform, and the band represents the standard deviation around the mean. 842 The bottom panel shows the distribution of the time from the trough to the peak for the 843 recorded waveforms. A threshold was set at $0.4 \mathrm{~ms}$ based on prior literature ((Guo et al., 844 2017), see Methods), for classifying the individual units into RSUs or FSUs.

846 Apparent in the aggregate PSTH is the expected higher baseline/ongoing activity and 847 sensory evoked responses in the FSUs compared to the RSUs, as shown in Figure 6B 848 ( $17 \mathrm{HZ}$ for FSU, $\mathrm{n}=32 ; \sim 3 \mathrm{~Hz}$ for $\mathrm{RSU}, \mathrm{n}=86$ ), along with several other observations 849 quantified in Figure 6C. As shown in the left panels of Figure 6C, in response to the 850 thalamic hyperpolarization (LED $\sim 17 \mathrm{~mW} / \mathrm{mm}^{2}$, double LED $\sim 35 \mathrm{~mW} / \mathrm{mm}^{2}$ ), there was a 851 relatively modest increase in baseline (pre-stimulus, $-50-0 \mathrm{~ms}$ ) firing rate for RSUs 
Borden et al., Thalamic state controls timing and synchronization of S1 cortex, 2021

852 (Control vs LED $p=5.3 e-4$, Control vs Double LED $p=0.012$, Wilcoxon signed-rank test, $853 \mathrm{n}=86$ ), while FSUs showed no significant change (Control vs LED $p=0.15$, Control vs 854 Double LED $p=0.30$, Wilcoxon signed-rank test, $n=32$ ). The response to the punctate 855 sensory stimulus was a relatively short latency, transient increase in firing activity in both 856 cell types, appearing to be relatively unchanged in overall magnitude with thalamic 857 hyperpolarization, further quantified in the middle panels of Figures $6 \mathrm{C}$ (early response, $8585-50 \mathrm{~ms}$ post stimulus). We observed a slight increase in the RSU evoked firing rate (less 859 than $\sim 10 \%$ increase, Control vs LED $p=0.067$, Control vs Double LED $p=0.0082$, 860 Wilcoxon signed-rank test, $\mathrm{n}=86$ ); however, FSUs displayed no statistical difference 861 (Control vs LED $p=0.30$, Control vs Double LED $p=0.45$, Wilcoxon signed-rank test, $862 \mathrm{n}=32$ ). This same effect was consistent across a larger range of optogenetic light levels 863 (Supplemental Figure S6).

Although the overall magnitude of the early phase of the sensory evoked response was relatively unchanged by the thalamic hyperpolarization for both the FSUs and RSUs, there was a qualitative change in shape of the PSTH, with an increased latency to peak in the early phase, and change in the later phase of the evoked response. Specifically, thalamic hyperpolarization induced a reduction in the later phase of the cortical response, and a dip below the baseline firing rate in both the FSUs and RSUs, as shown in the PSTHs in Figure 6B. This was reflected in a decrease in the late phase $(60-100 \mathrm{~ms})$ of the evoked response from the Control to the LED condition as shown in the right panels of Figure 6C (RSU - Control vs LED $p=0.017$, Control vs Double LED $p=0.014, n=86$, FSU Control vs LED $p=2.2 e-4$, Control vs Double LED $p=4.5 e-4, n=32$, Wilcoxon signed-rank test).

The changes in timing and shape of the PSTHs of the cortical neurons suggest the possibility that thalamic hyperpolarization could affect synchronization within the cortical network. We analyzed synchrony across simultaneously recorded cortical single-units. 880 Synchrony in firing across a pair of Neurons 1 and 2 is evaluated by examining the spike 881 times of Neuron 2 relative to a particular spike of Neuron 1, across all spikes of Neuron 882 1, as illustrated in Figure 7A (see Methods). This forms the spike cross-correlogram 883 (CCG), from which the synchrony is calculated as the integrated area within a +/- $7.5 \mathrm{~ms}$ 884 window (see Methods, (Wang et al., 2010; Whitmire et al., 2016)), as illustrated in the 885 bottom of Figure 7A. Due to the sensitivity of the synchrony metric to firing rate, we only 886 examined pairs with a robust measurement (more than 50 synchronous events) to control 887 for measurement accuracy (experimental results were invariant with different thresholds, 888 see Methods), which restricted the analysis to FSUs in our dataset. Figure 7B shows 889 rasters from an example pair of FSUs in the Control and double LED conditions (top), 890 along with the spikes identified to be synchronous by this criterion (bottom). Figure 7C 891 shows the aggregate cross-correlograms across 99 FSU pairs for the sensory evoked 
892 response, calculated from the spiking in the $100 \mathrm{~ms}$ window following the delivery of the sensory stimulus. There is an increase in the concentration of mass around 0 lag with 894 thalamic hyperpolarization, which is indicative of an increased synchrony. This is 895 summarized in Figure 7D, showing a significant increase across FSU units in the 896 measured synchrony (Control vs LED, $p=1.5 \mathrm{e}-8$, Control vs Double LED, $p=3.5 \mathrm{e}-13$, 897 Wilcoxon signed-rank test, $\mathrm{n}=99 \mathrm{FSU}$ pairs). For comparison, the synchrony was also 898 computed for the ongoing, spontaneous activity, revealing the synchronizing effect of the 899 transient sensory input. These results were qualitatively similar for various synchrony 900 window sizes (not shown).

901

\section{Modeling of the thalamic burst driven cortical E-I circuit} We next sought to understand the mechanistic basis of three key experimental results: 1) the invariant absolute S1 sensory response in the thalamic hyperpolarized (LED) condition in the awake mouse, despite the dramatic increase in sensory-evoked (burst) spikes in VPm; 2) the decrease in late (60 - 100 ms post-stimulus) spiking in the S1 response; and 3) the increase in sensory-evoked cortical synchrony in FS neurons in the thalamic hyperpolarized (LED) condition. To explore the potential role of various thalamic and cortical mechanisms, we constructed a simple model of the thalamocortical network, as described previously (Wright et al., 2021). This network mimics the numerical expansion of neurons at the thalamocortical junction, and incorporates several known properties of thalamocortical and intracortical connectivity (Figure 8A, see Methods and Supplemental Information on Cortical E-I Modeling). The network was composed of a single model VPm "barreloid" (40 independent spike trains) projecting to a single barrel "column", modeled as an interconnected network of 800 excitatory and 100 inhibitory single-compartment leaky integrate-and-fire neurons with clustered connectivity (LitwinKumar and Doiron, 2012; Bujan et al., 2015; Wright et al., 2017a, 2017b). For each VPm spike train, ongoing and sensory-evoked VPm tonic and burst spikes were drawn from inhomogeneous Poisson processes matching the empirical tonic and burst VPm PSTHs (Figure 8B, top two rows). Motivated by previous studies of cortical sensory adaptation in brain slice (Gabernet et al., 2005; Cruikshank et al., 2007b, 2010) and in anesthetized (Chung et al., 2002; Gabernet et al., 2005; Heiss et al., 2008; Cohen-Kashi Malina et al., 2013) and awake (Musall et al., 2014) rodents, we modeled rapid sensory adaptation of the thalamocortical (TC) synapse as instantaneous depression and exponential recovery of the TC synapse in response to a thalamic spike (see Methods). For a more detailed description of the model, see Supplemental Information on Cortical E-I Modeling, Section 1.

930

This model network succeeded in qualitatively reproducing the three results identified above: despite the fact that the VPm evoked rate in a 50 ms post-stimulus window was substantially boosted in the hyperpolarized (LED) condition, there was comparatively little 
932 change in the $\mathrm{S} 1$ excitatory and inhibitory early responses (Figure $8 \mathrm{~B}$, bottom two rows, 933 and Figure $8 \mathrm{C}$, left). In addition, late cortical spiking in the LED condition decreased 934 significantly compared to the Control condition (Figure 8B, insets in bottom two rows, and 935 Figure $8 \mathrm{C}$, right). Note that unlike the experimental data, synchrony measures from 936 simulated cortical activity were not limited by lack of firing events, enabling synchrony 937 measurements across both sub-populations. We found that the evoked pairwise 938 synchrony of $\mathrm{S} 1$ neurons was higher in the LED condition (Fig. 8D - G). In short, the 939 mechanisms incorporated in this model were sufficient to predict the key experimental 940 results. We next sought to identify the minimum set of mechanisms necessary to explain 941 each result (see Supplemental Information on Cortical E-I Modeling, Section 2). To do 942 this, we employed alternate versions of the model described above, and compared 943 simulation results across models to infer the role of each altered parameter.

945 First, we asked why the early absolute S1 response was invariant to thalamic 946 hyperpolarization, despite the boosted VPm response (Supplemental Information on 947 Cortical E-I Modeling, Section 2.1). We found that the overall increase in evoked VPm 948 rate in the LED condition yielded weaker TC synapses at the time of TC synaptic 949 transmission, due to activity-dependent synaptic depression (Supplemental Figure S7A, 950 B). Further, the short inter-spike intervals within bursts allowed little time between burst 951 spikes for synaptic recovery (Supplemental Figure S7C), and so the high proportion of 952 burst spikes in the LED condition further reduced the mean TC synaptic weight at the time 953 of synaptic transmission relative to the Control condition (Supplemental Figure S7D). 954 When either synaptic adaptation was removed, or the proportion of evoked burst spikes 955 was held fixed, the model S1 early response was instead boosted in the LED condition 956 compared to the Control condition (Supplemental Figure S7E). These results thus 957 demonstrate that the invariance of the early model response was due to more profound 958 TC synaptic adaptation induced by the substantial increase in thalamic bursting in the 959 LED condition. Notably, the S1 response was boosted in the LED condition in the 960 anesthetized condition, and the model also reproduced this result when we additionally 961 assumed that the LED-induced hyperpolarization enhanced sensory-evoked synchrony 962 across VPm units (Fig. S7F, G). Second, we asked why the late S1 response was 963 reduced in the LED condition compared to Control (Supplemental Information on Cortical 964 E-I Modeling, Section 2.2). We found that this reflected the late decrease in VPm firing, 965 as the overall time-course of cortical firing tracked that of VPm (Supplemental Figure S8A, 966 B). Finally, we asked why cortical pairwise synchrony increased in the LED condition 967 (Supplemental Information on Cortical E-I Modeling, Section 2.2). We found that the 968 change in the time-course of VPm firing yielded a higher concentration of cortical spikes 969 at intermediate response latencies, which shaped correlations between pairs of cortical 970 neurons; pairs of cortical spikes were more likely to be separated by short lags, and less 
bioRxiv preprint doi: https://doi.org/10.1101/2021.07.09.451656; this version posted July 10, 2021. The copyright holder for this preprint (which

was not certified by peer review) is the author/funder, who has granted bioRxiv a license to display the preprint in perpetuity. It is made available under aCC-BY-NC 4.0 International license.

Borden et al., Thalamic state controls timing and synchronization of S1 cortex, 2021

971 likely to be separated by longer lags, in the LED condition (Supplemental Figure S8C 972 F).

973

974 Taken together, our modeling results demonstrate the sensitivity of cortical sensory 975 representations to thalamic firing. The degree of thalamic bursting and rapid sensory 976 adaptation of TC synapses together determine the efficacy with which VPm spiking drives 977 S1. This predicts the counterintuitive result that enhanced VPm bursting does not result 978 in a boosted S1 response. At longer (tens of milliseconds) timescales, S1 activity levels 979 generally track that of VPm, and the late drop-out of VPm firing in the LED condition is 980 inherited by S1. Finally, the increased S1 synchrony in the LED condition reflects 981 changes in the overall time-course of VPm firing in the $100 \mathrm{~ms}$ following sensory stimulus 982 onset. 
bioRxiv preprint doi: https://doi.org/10.1101/2021.07.09.451656; this version posted July 10, 2021. The copyright holder for this preprint (which

was not certified by peer review) is the author/funder, who has granted bioRxiv a license to display the preprint in perpetuity. It is made available under aCC-BY-NC 4.0 International license.

Borden et al., Thalamic state controls timing and synchronization of S1 cortex, 2021

984

985

986

987

988

989

990

991

992

993

994

995

996

997

998

999

1000

1001

1002

1003

1004

1005

1006

1007

1008

1009

1010

1011

1012

1013

1014

1015

1016

1017

1018

1019

1020

1021

1022

1023

\section{DISCUSSION}

The sensory thalamus controls the flow of signaling from the periphery to cortex, ultimately gating what we do and do not perceive about the outside world. Despite its critical role in sensing, how this circuit controls signaling remains poorly understood. Here, through a range of experimental approaches in the awake, head-fixed mouse, we show that manipulation of thalamic state through hyperpolarization switches the thalamus from a tonic to burst firing mode that serves to amplify thalamic sensory evoked responses. Sensory cortex, on the other hand, exhibits an invariant absolute evoked response despite the increased thalamic input, instead demonstrating increased timing precision, a focusing of spatial activation, and increased synchrony of spiking. Thalamocortical network modeling further reveals that the induced changes in thalamic spike timing and the engagement of thalamocortical synaptic depression by enhanced bursting are sufficient to explain the increase in cortical synchronization and the invariant cortical response amplitude, respectively. The findings here present a highly sensitive, statedependent timing-based gating of sensory signaling to cortex.

\section{Thalamic Gating and the Effects on Cortex}

Given the amplification of sensory evoked thalamic responses in thalamus with thalamic hyperpolarization, the effects on cortex were surprising. The absolute cortical sensory evoked response was invariant to thalamic hyperpolarization in awake mice, despite the boosted thalamic response. Note that the increase in cortical background firing rate and the invariant absolute sensory evoked cortical response amplitude together led to a decrease in the cortical response relative to baseline, which was consistently reflected in both the relative single unit (Figure 4J) and GEVI (Figure 4G) measures. What was enhanced by the thalamic hyperpolarization, however, was instead the timing precision and synchrony of the sensory evoked cortical activity, which are important to understand in terms of the mechanisms underlying this observation and the potential implications for downstream signaling. The modeling results suggest an interplay between the timing related changes in thalamic firing with a shift to burst mode, and the timing dependent depression of thalamocortical synapses, are likely the key role players in the observed dynamics, although more extensive experiments involving causal manipulations of these mechanisms would need to be conducted to more conclusively establish this explanation. Moreover, the enhanced timing and synchrony of the sensory evoked S1 response suggests a potent input for downstream signaling, despite the invariance in firing rate, but corresponding recordings of the $\mathrm{S} 1$ recipient regions would be needed to ultimately determine the effect of the changes in S1. Finally, neurons in VPm thalamus sends axonal projections differentially across laminae in S1 (Sermet et al., 2019), likely resulting in variations in the effects of thalamic hyperpolarization on $\mathrm{S} 1$ neurons in different cortical layers. Layer specific effects were not targeted in this study, and thus electrode 
1024 recordings obtained across laminae were combined. It is likely that the thalamic-timing 1025 related effects on S1 activity we report here would be more pronounced in thalamic 1026 recipient layers of $\mathrm{S} 1$ as compared to layers not receiving direct monosynaptic thalamic 1027 input, resulting in a more variable effect in our pooled data. In contrast to the electrode 1028 recordings, the optical GEVI imaging approach utilized here captures primarily sub1029 threshold activity of S1 neurons with cell bodies in layer 2/3 (Petersen et al., 2003), which 1030 could explain the more dramatic effects seen in the voltage imaging, although the imaging 1031 obscures any differences across excitatory and inhibitory sub-populations.

1032

1033

\section{Invariance in Baseline Thalamic Firing Activity}

1034 In this study, we utilized optogenetic hyperpolarization to bias thalamic sensory 1035 responses toward bursting, without significantly changing other dynamics that might 1036 indirectly impact sensory responses. One particularly surprising finding here was that 1037 despite optogenetically induced hyperpolarization of thalamus, the overall baseline firing 1038 rate of thalamic neurons was not suppressed, but instead unchanged. Following a 1039 transient decrease in firing rate, the recorded VPm neurons returned to their original 1040 baseline firing rate. This finding is, however, consistent with other reported observations. 1041 In the visual pathway for example, optogenetic excitation of the reticular nucleus (TRN) 1042 transiently silences LGN, followed by a return to the original firing rate at steady-state 1043 (Reinhold et al., 2015). In the somatosensory pathway, strong photoinhibition attenuated 1044 VPm thalamic firing rate, but failed to quench activity altogether (Halassa et al., 2011; 1045 Poulet et al., 2012a; Lewis et al., 2015; Reinhold et al., 2015; Yu et al., 2016). The most 1046 parsimonious explanation given the observations here is that moderate amounts of 1047 hyperpolarization serve to engage the dynamics of the T-type calcium channels that are 1048 inactive at normal baseline conditions, effectively compensating for the loss in tonic 1049 spiking due to the hyperpolarization. Although the optogenetic approach here does not 1050 enable direct observation of the magnitude of the hyperpolarizing input, separate 1051 intracellular in-vitro slice experiments where we repeated the protocol while patching on 1052 to VPm neurons revealed relatively modest amounts of hyperpolarization that were well1053 sustained during constant light illumination. This is important, as recent studies have 1054 suggested that prolonged activation of specific opsins can have unintended 1055 consequences, notably here the possibility of changes in the reversal potential for chloride 1056 (Raimondo et al., 2012b), which could theoretically result in changes in the degree of 1057 hyperpolarization, although halorhodopsin as a pump is less directly affected by 1058 immediate (local) changes in reversal potential as compared to channel-based 1059 optogenetics. Further, with halorhodopsin there is the potential for photoinactivation and 1060 decreased photocurrents (Zhang et al., 2019), which could also change the degree of 1061 hyperpolarization over time. Through the combination of the intracellular control 1062 experiments and replication of the primary result through the integrate and fire or burst 
bioRxiv preprint doi: https://doi.org/10.1101/2021.07.09.451656; this version posted July 10, 2021. The copyright holder for this preprint (which

was not certified by peer review) is the author/funder, who has granted bioRxiv a license to display the preprint in perpetuity. It is made available under aCC-BY-NC 4.0 International license.

Borden et al., Thalamic state controls timing and synchronization of S1 cortex, 2021

1063 (IFB) model, the likelihood that these possible effects played a primary role here is low, 1064 especially over the relatively short timescales shown here.

1065

1066

Importantly, beyond the engagement of the T-type calcium channel burst mechanism

1067 upon initial hyperpolarization, it would seem that additional hyperpolarization would further push the neuron away from threshold, making it more difficult to burst, which would predict a corresponding decrease in firing rate. However, we found that increased hyperpolarization increased the number of spikes per burst, which served to offset the decrease in the number of bursts with increasing hyperpolarization. The result is a surprisingly resilient mechanism in response to this perturbation. Although other elements of the circuit certainly play a role in this observation (e.g., TRN), the findings here suggest that the intrinsic properties of the thalamic neurons could be a primary mechanism at work.

1076

1077

1078

1079

1080

1081

1082

1083

1084

1085

1086

1087

1088

1089

1090

1091

1092

1093

1094

1095

1096

1097

1098

1099

1100

1101

1102

1103

\section{Thalamic Manipulation and Intrinsic Modulation}

The optogenetic manipulation approach here was utilized to gain systematic control over thalamic burst/tonic firing modes to precisely quantify the functional effects at the level of thalamus and primary sensory cortex. This is especially important in the awake, unanesthetized brain, where continuous fluctuations in thalamic state preclude a clear picture of the causal effects of this mechanism in the thalamocortical circuit. It is nevertheless important to place the findings here in the context of naturally occurring fluctuations in thalamic state. Ongoing thalamic spontaneous bursting events were traditionally only associated with slow wave sleep or anesthesia; however, this has been largely disproven, with low rates of bursts occurring both spontaneously and during naturalistic stimuli in awake somatosensory (Swadlow and Gusev, 2001a; Stoelzel et al., 2009; Wright et al., 2021), visual (Niell and Stryker, 2011), and auditory systems (Massaux et al., 2004). The thalamus receives a range of complex, excitatory and inhibitory inputs that interact with the intrinsic cell properties to collectively set the baseline membrane potential and firing rate of these neurons in normal physiological conditions. In the experiments presented here, the mice were awake and head-fixed, but otherwise idling, trained to tolerate the head-fixation with the periodic delivery of a water reward. During the experiments described here, it appeared that the animals remained engaged and were unlikely to spend much time in a state of low arousal, setting the baseline firing rate and level of neuromodulatory input to the thalamus to a corresponding level. Although the optogenetic manipulation here is likely quite strong relative to other modulatory inputs to thalamus, it is true that these other inputs could affect and influence the thalamus in concert with our manipulation. However, any effects from this would likely be averaged out across trials, only contributing to the variability of the measures we present. It is also the case that cortex is under the influence of a range of modulatory inputs, some of which are shared with thalamus, and thus our manipulation of thalamic 
bioRxiv preprint doi: https://doi.org/10.1101/2021.07.09.451656; this version posted July 10, 2021. The copyright holder for this preprint (which

was not certified by peer review) is the author/funder, who has granted bioRxiv a license to display the preprint in perpetuity. It is made available under aCC-BY-NC 4.0 International license.

Borden et al., Thalamic state controls timing and synchronization of S1 cortex, 2021

1104 state could effectively serve to decouple the two regions, resulting in the thalamus and 1105 cortex being in different "states", a condition which is likely not entirely physiological. 1106 However, there is strong evidence that thalamic activity itself is a strong driver of cortical 1107 state (Poulet et al., 2012a), and thus the manipulations of thalamic state could indirectly 1108 gain control of cortical state as well. To really disentangle these issues of brain state 1109 across different structures like the thalamus and cortex, it is likely that approaches such 1110 as closed-loop feedback control of neural activity (Bolus et al., 2018, 2021) need to be 1111 employed to more strongly and effectively explore the coupling across brain regions.

1112

\section{Potential Role in Sensory Signaling}

1114 Almost four decades ago, Crick proposed a provocative hypothesis - that the thalamic1115 reticular complex serves as a dynamic gate for attentional control of sensory signaling to 1116 cortex (Crick, 1984). Further refinement of this idea suggested that the switching between 1117 tonic and burst firing modes of thalamic neurons that is facilitated by the unique dynamics 1118 of the T-type calcium channels that are prevalent in the thalamus could establish a context dependent signaling (Sherman and Guillery, 2002; Sherman, 2005). In this framework, burst spiking would promote the detection of salient sensory features, while tonic spiking would promote the transmission of details about the nature of the sensory stimulus. Furthermore, the thalamic burst would also potentially provide a "wake-up call" to cortex (Sherman, 2001), garnering attentional resources that ultimately would serve to switch the thalamic mode of firing to tonic through depolarizing corticothalamic feedback. As attractive a framework as this is, it is also a daunting theory to test experimentally due to the complexity of the circuit, the required specificity of recording and manipulation, and ultimately the need to cast in the context of changing states of arousal during trained behaviors. Several studies in the anesthetized brain have shown that thalamic bursting is indeed well driven by the appearance of salient sensory features (Lesica and Stanley, 2004; Alitto et al., 2005; Denning and Reinagel, 2005), that this promotes the detection of change in the sensory input from the perspective of an ideal observer of thalamic spiking (Lesica and Stanley, 2004), and that this sensitivity is strongly shaped by thalamic state (Lesica et al., 2006). Further studies showing the sensitivity of sensory cortex to thalamic bursting (Swadlow and Gusev, 2001) seem to set the stage for at least part of the overarching coding scheme in which case the cortical response would be amplified by the thalamic bursts in "detect" mode, yet none of this has been tested in the awake brain to date. Although the results here in the awake brain demonstrate that the absolute S1 sensory evoked response was invariant to thalamic hyperpolarization and even diminished relative to background cortical activity, despite potent sensory-evoked thalamic bursting, what emerges is increased timing precision, increased focus of spatial activation, and a corresponding synchronization of the cortical sensory-evoked response. Given the likely timing sensitivity of down-stream brain structures in the sensorimotor arc, the synchronization of cortical activity may in fact be a more critical element of cortical 
bioRxiv preprint doi: https://doi.org/10.1101/2021.07.09.451656; this version posted July 10,2021. The copyright holder for this preprint (which

was not certified by peer review) is the author/funder, who has granted bioRxiv a license to display the preprint in perpetuity. It is made available under aCC-BY-NC 4.0 International license.

Borden et al., Thalamic state controls timing and synchronization of S1 cortex, 2021

1144 signaling than overall magnitude, supported by recent behavioral work demonstrating the 1145 importance of cortical synchrony over firing rate (Jadhav et al., 2009). Taken together, 1146 the results here point to timing rather than response magnitude as a fundamental 1147 currency of the thalamocortical circuit, presenting a dynamic, state-dependent timing1148 based gating of sensory signaling to cortex that has strong implications for detectability 1149 and discriminability in complex sensory environments. 
bioRxiv preprint doi: https://doi.org/10.1101/2021.07.09.451656; this version posted July 10, 2021. The copyright holder for this preprint (which

was not certified by peer review) is the author/funder, who has granted bioRxiv a license to display the preprint in perpetuity. It is made available under aCC-BY-NC 4.0 International license.

Borden et al., Thalamic state controls timing and synchronization of S1 cortex, 2021

\section{References}

1156 Aasebø IEJ, Lepperød ME, Stavrinou M, Nøkkevangen S, Einevoll G, Hafting T, Fyhn M (2017) Temporal Processing in the Visual Cortex of the Awake and Anesthetized Rat. Eneuro 4:ENEURO.0059-17.2017.

Aguilar JR, Castro-Alamancos M a (2005) Spatiotemporal gating of sensory inputs in thalamus during quiescent and activated states. J Neurosci 25:10990-11002.

Alitto HJ, Weyand TG, Usrey WM (2005) Distinct properties of stimulus-evoked bursts in the lateral geniculate nucleus. J Neurosci 25:514-523.

Béhuret S, Deleuze C, Bal T (2015) Corticothalamic Synaptic Noise as a Mechanism for Selective Attention in Thalamic Neurons. Front Neural Circuits 9:1-21.

Bolus MF, Willats AA, Rozell CJ, Stanley GB (2021) State-space optimal feedback control of optogenetically driven neural activity. J Neural Eng 18:036006.

Bolus MF, Willats AA, Whitmire CJ, Rozell CJ, Stanley GB (2018) Design strategies for dynamic closed-loop optogenetic neurocontrol in vivo. J Neural Eng 15:026011.

Borden PY, Ortiz AD, Waiblinger C, Sederberg AJ, Morrissette AE, Forest CR, Jaeger D, Stanley GB (2017) Genetically expressed voltage sensor ArcLight for imaging large scale cortical activity in the anesthetized and awake mouse. Neurophotonics 4:031212.

Brecht M, Sakmann B (2002) Whisker maps of neuronal subclasses of the rat ventral posterior medial thalamus, identified by whole-cell voltage recording and morphological reconstruction. J Physiol:495-515.

Bruno RM, Simons DJ (2002) Feedforward mechanisms of excitatory and inhibitory cortical receptive fields. J Neurosci 22:10966-10975.

Bujan AF, Aertsen A, Kumar XA, Kumar A, Kumar XA (2015) Role of Input Correlations in Shaping the Variability and Noise Correlations of Evoked Activity in the Neocortex. J Neurosci 35:8611-8625.

Castro-Alamancos MA (2002) Different temporal processing of sensory inputs in the rat thalamus during quiescent and information processing states in vivo. J Neurophysiol 87:946-953.

Chung S, Li X, Nelson SB (2002) Short-term depression at thalamocortical synapses contributes to rapid adaptation of cortical sensory responses in vivo. Neuron 34:437-446.

Cohen-Kashi Malina K, Jubran M, Katz Y, Lampl I (2013) Imbalance between excitation and inhibition in the somatosensory cortex produces postadaptation facilitation. J Neurosci 33:8463-8471.

Crandall SR, Cruikshank SJ, Connors BW (2015a) A Corticothalamic Switch: Controlling the Thalamus with Dynamic Synapses. Neuron 86:768-782.

Crandall SRR, Cruikshank SJJ, Connors BWW, Crandall SRR, Cruikshank SJJ, Connors BWW (2015b) A Corticothalamic Switch: Controlling the Thalamus with Dynamic Synapses. Neuron 86:1-15.

Crick F (1984) Function of the thalamic reticular complex: The searchlight hypothesis. Proc Natl Acad Sci U S A 81:4586-4590.

Cruikshank SJ, Lewis TJ, Connors BW (2007a) Synaptic basis for intense thalamocortical activation of feedforward inhibitory cells in neocortex. Nat Neurosci 10:462-468.

Cruikshank SJ, Lewis TJ, Connors BW (2007b) Synaptic basis for intense thalamocortical activation of feedforward inhibitory cells in neocortex. Nat Neurosci 10:462-468. 
bioRxiv preprint doi: https://doi.org/10.1101/2021.07.09.451656; this version posted July 10, 2021. The copyright holder for this preprint (which

was not certified by peer review) is the author/funder, who has granted bioRxiv a license to display the preprint in perpetuity. It is made available under aCC-BY-NC 4.0 International license.

Borden et al., Thalamic state controls timing and synchronization of S1 cortex, 2021

Cruikshank SJ, Urabe H, Nurmikko A V., Connors BW (2010) Pathway-Specific Feedforward Circuits between Thalamus and Neocortex Revealed by Selective Optical Stimulation of Axons. Neuron 65:230-245.

Denning KS, Reinagel P (2005) Visual control of burst priming in the anesthetized lateral geniculate nucleus. J Neurosci 25:3531-3538.

1204

Fogerson PM, Huguenard JR (2016) Tapping the Brakes: Cellular and Synaptic Mechanisms that Regulate Thalamic Oscillations. Neuron 92:687-704.

1206

Gabernet L, Jadhav SP, Feldman DE, Carandini M, Scanziani M (2005) Somatosensory integration controlled by dynamic thalamocortical feed-forward inhibition. Neuron 48:315-327.

Gentet LJ, Avermann M, Matyas F, Staiger JF, Petersen CCHH (2010) Membrane potential dynamics of GABAergic neurons in the barrel cortex of behaving mice. Neuron 65:422-435.

Godwin DW, Vaughan JW, Sherman SM (1996) Metabotropic glutamate receptors switch visual 76:1800-1816.

1215

1216

Greenberg DS, Houweling AR, Kerr JND (2008) Population imaging of ongoing neuronal activity in the visual cortex of awake rats. Nat Neurosci 11:749-751.

Guo W, Clause AR, Barth-Maron A, Polley DB (2017) A Corticothalamic Circuit for Dynamic Switching between Feature Detection and Discrimination. Neuron 95:180-194.e5.

Haider B, Häusser M, Carandini M (2013) Inhibition dominates sensory responses in the awake cortex. Nature 493:97-100.

Halassa MM, Acsády L (2016) Thalamic Inhibition: Diverse Sources, Diverse Scales. Trends Neurosci 39:680-693.

Halassa MM, Siegle JH, Ritt JT, Ting JT, Feng G, Moore Cl (2011) Selective optical drive of thalamic reticular nucleus generates thalamic bursts and cortical spindles. Nat Neurosci 14:1118-1120.

Heiss JE, Katz Y, Ganmor E, Lampl I (2008) Shift in the Balance between Excitation and Inhibition during Sensory Adaptation of S1 Neurons. J Neurosci 28:13320-13330.

Hirata A, Castro-Alamancos MA (2010) Neocortex network activation and deactivation states controlled by the Thalamus. J Neurophysiol 103:1147-1157.

Huguenard JR, McCormick D a. (2007) Thalamic synchrony and dynamic regulation of global forebrain oscillations. Trends Neurosci 30:350-356.

Jadhav SP, Wolfe J, Feldman DE (2009) Sparse temporal coding of elementary tactile features during active whisker sensation. Nat Neurosci 12:792-800.

Jin L, Han Z, Platisa J, Wooltorton JRA, Cohen LB, Pieribone VA (2012) Single Action Potentials and Subthreshold Electrical Events Imaged in Neurons with a Fluorescent Protein Voltage Probe. Neuron 75:779-785.

Khatri V, Hartings JA, Simons DJ (2004) Adaptation in thalamic barreloid and cortical barrel neurons to periodic whisker deflections varying in frequency and velocity. J Neurophysiol 92:3244-3254.

Kim HR, Hong SZ, Fiorillo CD (2015) T-type calcium channels cause bursts of spikes in motor but not sensory thalamic neurons during mimicry of natural patterns of synaptic input. Front Cell Neurosci 9:428.

Kimura F, Itami C, Ikezoe K, Tamura H, Fujita I, Yanagawa Y, Obata K, Ohshima M (2010a) Fast 
bioRxiv preprint doi: https://doi.org/10.1101/2021.07.09.451656; this version posted July 10, 2021. The copyright holder for this preprint (which

was not certified by peer review) is the author/funder, who has granted bioRxiv a license to display the preprint in perpetuity. It is made available under aCC-BY-NC 4.0 International license.

Borden et al., Thalamic state controls timing and synchronization of S1 cortex, 2021

1243

1244

1245

1246

1247

1248

1249

1250

1251

1252

1253

1254

1255

1256

1257

1258

1259

1260

1261

1262

1263

1264

1265

1266

1267

1268

1269

1270

1271

1272

1273

1274

1275

1276

1277

1278

1279

1280

1281

1282

1283

1284

1285

1286

activation of feedforward inhibitory neurons from thalamic input and its relevance to the regulation of spike sequences in the barrel cortex. J Physiol 588:2769-2787.

Kimura F, Itami C, Ikezoe K, Tamura H, Fujita I, Yanagawa Y, Obata K, Ohshima M (2010b) Fast activation of feedforward inhibitory neurons from thalamic input and its relevance to the regulation of spike sequences in the barrel cortex. J Physiol 588:2769-2787.

Kirchgessner MA, Franklin AD, Callaway EM (2020) Context-dependent and dynamic functional influence of corticothalamic pathways to first- and higher-order visual thalamus. Proc Natl Acad Sci 117:13066-13077.

Lesica N a, Stanley GB (2004) Encoding of natural scene movies by tonic and burst spikes in the lateral geniculate nucleus. J Neurosci 24:10731-10740.

Lesica N a, Weng C, Jin J, Yeh C-I, Alonso J-M, Stanley GB (2006) Dynamic encoding of natural luminance sequences by LGN bursts. PLoS Biol 4:e209.

Lewis LD, Voigts J, Flores FJ, Ian Schmitt L, Wilson MA, Halassa MM, Brown EN (2015) Thalamic reticular nucleus induces fast and local modulation of arousal state. Elife 4:1-23.

Li L, Ebner FF (2016) Cortex dynamically modulates responses of thalamic relay neurons through prolonged circuit-level disinhibition in rat thalamus in vivo. J Neurophysiol 116:2368-2382.

Litwin-Kumar A, Doiron B (2012) Slow dynamics and high variability in balanced cortical networks with clustered connections. Nat Neurosci 15:1498-1505.

Lustig BR, Friedman RM, Winberry JE, Ebner FF, Roe AW (2013) Voltage-sensitive dye imaging reveals shifting spatiotemporal spread of whisker-induced activity in rat barrel cortex. J Neurophysiol 109:2382-2392.

Ma Y, Shaik MA, Kim SH, Kozberg MG, Thibodeaux DN, Zhao HT, Yu H, Hillman EMC (2016) Wide-field optical mapping of neural activity and brain haemodynamics: considerations and novel approaches. Philos Trans R Soc B Biol Sci 371:20150360.

Massaux A, Dutrieux G, Cotillon-Williams N, Manunta Y, Edeline J-M (2004) Auditory Thalamus Bursts in Anesthetized and Non-Anesthetized States: Contribution to Functional Properties. J Neurophysiol 91:2117-2134.

McCormick DA, Connors BW, Lighthall JW, Prince DA (1985) Comparative electrophysiology of pyramidal and sparsely spiny stellate neurons of the neocortex. J Neurophysiol 54:782806.

McCormick DA, von Krosigk M (1992) Corticothalamic activation modulates thalamic firing through glutamate "metabotropic" receptors. Proc Natl Acad Sci 89:2774-2778.

Mease RA, Krieger P, Groh A (2014) Cortical control of adaptation and sensory relay mode in the thalamus. Proc Natl Acad Sci 111:6798-6803.

Millard DC, Whitmire CJ, Gollnick CA, Rozell CJ, Stanley GB (2015) Electrical and Optical Activation of Mesoscale Neural Circuits with Implications for Coding. J Neurosci 35:1570215715.

Murray Sherman S (2001) Tonic and burst firing: Dual modes of thalamocortical relay. Trends Neurosci 24:122-126.

Musall S et al. (2014) Tactile frequency discrimination is enhanced by circumventing neocortical adaptation. Nat Neurosci 17:1567-1573.

Niell CM, Stryker MP (2010) Modulation of visual responses by behavioral state in mouse visual cortex. Neuron 65:472-479. 
bioRxiv preprint doi: https://doi.org/10.1101/2021.07.09.451656; this version posted July 10, 2021. The copyright holder for this preprint (which

was not certified by peer review) is the author/funder, who has granted bioRxiv a license to display the preprint in perpetuity. It is made available under aCC-BY-NC 4.0 International license.

Borden et al., Thalamic state controls timing and synchronization of S1 cortex, 2021

1287

1288

1289

1290

1291

1292

1293

1294

1295

1296

1297

1298

1299

1300

1301

1302

1303

1304

1305

1306

1307

1308

1309

1310

1311

1312

1313

1314

1315

1316

1317

1318

1319

1320

1321

1322

1323

1324

1325

1326

1327

1328

1329

1330

Niell CM, Stryker MP (2011) Modulation of visual responses by behavioral state in mouse visual cortex. Neuron 65:472-479.

Olsen SR, Bortone DS, Adesnik H, Scanziani M (2012) Gain control by layer six in cortical circuits of vision. Nature 483:47-52.

Owen SF, Liu MH, Kreitzer AC (2019) Thermal constraints on in vivo optogenetic manipulations. Nat Neurosci 22:1061-1065.

Petersen CCH, Grinvald A, Sakmann B (2003) Spatiotemporal dynamics of sensory responses in layer $2 / 3$ of rat barrel cortex measured in vivo by voltage-sensitive dye imaging combined with whole-cell voltage recordings and neuron reconstructions. J Neurosci 23:1298-1309.

Pinto DJ, Brumberg JC, Simons DJ (2000) Circuit dynamics and coding strategies in rodent somatosensory cortex. J Neurophysiol 83:1158-1166.

Poulet JF a, Fernandez LMJ, Crochet S, Petersen CCH (2012a) Thalamic control of cortical states. Nat Neurosci 15:370-372.

Poulet JFA, Fernandez LMJ, Crochet S, Petersen CCH (2012b) Thalamic control of cortical states. Nat Neurosci 15:370-372.

Raimondo J V., Kay L, Ellender TJ, Akerman CJ (2012a) Optogenetic silencing strategies differ in their effects on inhibitory synaptic transmission. Nat Neurosci.

Raimondo J V., Kay L, Ellender TJ, Akerman CJ (2012b) Optogenetic silencing strategies differ in their effects on inhibitory synaptic transmission. Nat Neurosci 15:1102-1104.

Reinhold K, Lien AD, Scanziani M (2015) Distinct recurrent versus afferent dynamics in cortical visual processing. Nat Neurosci 18:1789-1797.

Rodenkirch C, Liu Y, Schriver BJ, Wang Q (2019) Locus coeruleus activation enhances thalamic feature selectivity via norepinephrine regulation of intrathalamic circuit dynamics. Nat Neurosci 22:120-133.

Sermet BS, Truschow P, Feyerabend M, Mayrhofer JM, Oram TB, Yizhar O, Staiger JF, Petersen $\mathrm{CCH}$ (2019) Pathway-, layer- and cell-type-specific thalamic input to mouse barrel cortex. Elife 8:1-28.

Sherman SM (2001) A wake-up call from the thalamus. Nat Neurosci 4:344-346.

Sherman SM (2005) Thalamic relays and cortical functioning. In: Progress in Brain Research, pp 107-126.

Sherman SM, Guillery RW (2002) The role of the thalamus in the flow of information to the cortex Adams P, Guillery RW, Sherman SM, Sillito AM, eds. Philos Trans R Soc London Ser B Biol Sci 357:1695-1708.

Smith GD, Cox CL, Sherman SM, Rinzel J (2000) Fourier analysis of sinusoidally driven thalamocortical relay neurons and a minimal integrate-and-fire-or-burst model. J Neurophysiol 83:588-610.

Speed A, Del Rosario J, Burgess CP, Haider B (2019) Cortical State Fluctuations across Layers of V1 during Visual Spatial Perception. Cell Rep 26:2868-2874.e3.

Stanley GB, Jin J, Wang Y, Desbordes G, Wang Q, Black MJ, Alonso J-MJ-M (2012) Visual orientation and directional selectivity through thalamic synchrony. J Neurosci 32:90739088.

Stoelzel CR, Bereshpolova Y, Swadlow H a (2009) Stability of Thalamocortical Synaptic Transmission across Awake Brain States. J Neurosci 29:6851-6859.

Stujenske JM, Spellman T, Gordon JA (2015) Modeling the Spatiotemporal Dynamics of Light 
bioRxiv preprint doi: https://doi.org/10.1101/2021.07.09.451656; this version posted July 10, 2021. The copyright holder for this preprint (which

was not certified by peer review) is the author/funder, who has granted bioRxiv a license to display the preprint in perpetuity. It is made available under aCC-BY-NC 4.0 International license.

Borden et al., Thalamic state controls timing and synchronization of S1 cortex, 2021

1331

1332

1333

1334

1335

1336

1337

1338

1339

1340

1341

1342

1343

1344

1345

1346

1347

1348

1349

1350

1351

1352

1353

1354

1355

1356

1357

1358

1359

1360

1361

1362

1363

1364

1365

1366

1367

1368

1369

1370

1371

1372

1373

1374

and Heat Propagation for In Vivo Optogenetics. Cell Rep 12:525-534.

Suzuki M, Larkum ME (2020) General Anesthesia Decouples Cortical Pyramidal Neurons. Cell 180:666-676.e13.

Suzuki S, Rogawski M a (1989) T-type calcium channels mediate the transition between tonic and phasic firing in thalamic neurons. Proc Natl Acad Sci U S A 86:7228-7232.

Swadlow H a, Gusev a G (2001) The impact of "bursting" thalamic impulses at a neocortical synapse. Nat Neurosci 4:402-408.

Swadlow HA. (2002) Thalamocortical control of feed-forward inhibition in awake somatosensory 'barrel' cortex Adams P, Guillery RW, Sherman SM, Sillito AM, eds. Philos Trans R Soc London Ser B Biol Sci 357:1717-1727.

Swadlow HA, Bezdudnaya T, Gusev AG (2005) Spike timing and synaptic dynamics at the awake thalamocortical synapse. Prog Brain Res 149:91-105.

Taub AH, Katz Y, Lampl I (2013) Cortical Balance of Excitation and Inhibition Is Regulated by the Rate of Synaptic Activity. J Neurosci 33:14359-14368.

Urbain N, Salin PA, Libourel PA, Comte JC, Gentet L, Petersen CCHH (2015) Whisking-Related Changes in Neuronal Firing and Membrane Potential Dynamics in the Somatosensory Thalamus of Awake Mice. Cell Rep 13:647-656.

Varela C (2014) Thalamic neuromodulation and its implications for executive networks. Front Neural Circuits 8:1-22.

Vizuete JA, Pillay S, Diba K, Ropella KM, Hudetz AG (2012) Monosynaptic functional connectivity in cerebral cortex during wakefulness and under graded levels of anesthesia. Front Integr Neurosci 6:1-11.

Wang Q, Webber RM, Stanley GB (2010) Thalamic synchrony and the adaptive gating of information flow to cortex. Nat Neurosci 13:1534-1541.

Whitmire, CJ, Liew YJ, Stanley GB. Thalamic state influences timing precision in the thalamocortical circuit, J. Neurophysiol., 125(5):1833-1850, 2021.

Whitmire CJ, Waiblinger C, Schwarz C, Stanley GB (2016) Information Coding through Adaptive Gating of Synchronized Thalamic Bursting. Cell Rep 14:795-807.

Wright NC, Borden PY, Liew YJ, Bolus MF, Stoy WM, Forest CR, Stanley GB (2021) Rapid Cortical Adaptation and the Role of Thalamic Synchrony During Wakefulness. J Neurosci:JN-RM3018-20.

Wright NC, Hoseini MS, Wessel R (2017a) Adaptation modulates correlated subthreshold response variability in visual cortex. J Neurophysiol 118:1257-1269.

Wright NC, Hoseini MS, Yasar TB, Wessel R (2017b) Coupling of synaptic inputs to local cortical activity differs among neurons and adapts after stimulus onset. J Neurophysiol 118:33453359.

Yang H, Kwon SE, Severson KS, O'Connor DH (2016) Origins of choice-related activity in mouse somatosensory cortex. Nat Neurosci 19:127-134.

Yu J, Gutnisky DA, Hires SA, Svoboda K (2016) Layer 4 fast-spiking interneurons filter thalamocortical signals during active somatosensation. Nat Neurosci 19:1-14.

Yu J, Hu H, Agmon A, Svoboda K (2019) Recruitment of GABAergic Interneurons in the Barrel Cortex during Active Tactile Behavior. Neuron 104:412-427.e4.

Zhang C, Yang S, Flossmann T, Gao S, Witte OW, Nagel G, Holthoff K, Kirmse K. Optimized photo-stimulation of halorhodopsin for long-term neuronal inhibition. BMC Biol. 2019 Nov 
bioRxiv preprint doi: https://doi.org/10.1101/2021.07.09.451656; this version posted July 10, 2021. The copyright holder for this preprint (which

was not certified by peer review) is the author/funder, who has granted bioRxiv a license to display the preprint in perpetuity. It is made available under aCC-BY-NC 4.0 International license.

Borden et al., Thalamic state controls timing and synchronization of S1 cortex, 2021

27;17(1):95. doi: 10.1186/s12915-019-0717-6.

1376

1377

1378

1379

1380

1381

1382

1383

1384

1385

1386

1387

1388

1389

1390

1391

1392

1393

1394

1395

1396

1397

1398

1399

1400

1401

1402

1403

1404

1405

1406

1407

1408

1409

1410

1411

1412

1413

1414

1415

1416

1417

1418

\section{FIGURE CAPTIONS}

Figure 1. Thalamic hyperpolarization amplifies direct feedforward input from thalamus in the anesthetized mouse. A. Pathway of the mouse vibrissa system from the facial vibrissae in the periphery, to brainstem, to thalamus, to S1. B. Experimental setup. Mice were injected with the viral vector eNphR3.0 (AAV5-CamlIKianse-eNphR3.0-mCherry) targeting the ventral posteriormedial (VPm) region of the thalamus and the viral vector ArcLight (AAV1-hysn1- ArcLightD- SV40) in the vibrissa region of the primary somatosensory cortex (S1). For optogenetic hyperpolarization, thalamic units were presented with constant illumination (LED, $590 \mathrm{~nm}$, approx. $17 \mathrm{~mW} / \mathrm{mm}^{2}$ ) from a $200 \mu \mathrm{m}$ optic fiber, with LED illumination starting at $0.5 \mathrm{~s}$ preceding stimulus $(t=-0.5 \mathrm{~s})$ and ending $0.5 \mathrm{~s}$ after stimulus $(\mathrm{t}=0.5)$, while recording simultaneously with a single tungsten electrode. Note that unlike for the awake recordings, light presentation was not ramped. For cortical GEVI imaging, the entire cortical area was illuminated through the thinned skull at $465 \mathrm{~nm}$ with an LED and imaged with a CCD imaging setup (see Methods). C. Thalamic bursts were identified as two or more spikes with inter-spike interval (ISIs) less than $4 \mathrm{ms,}$ preceded by silence for $100 \mathrm{~ms}$ or more. Thalamic spikes that were part of an identified burst were classified as putative burst spikes (red), and all else were classified as tonic spikes (black). D. Peri-stimulus time histogram (PSTH) for thalamic spiking in response to a single, punctate whisker stimulus $(t=0)$ for the Control (no light, top panel) and thalamic hyperpolarized (LED, bottom panel) conditions, 29 units. Instantaneous firing rates (bin size $2 \mathrm{~ms}$ ) shown for all spikes (black) and putative burst spikes (red). E. Left Mean sensory-evoked thalamic firing rate ( $n=29$ thalamic units) over the $0-30 \mathrm{~ms}$ time window increased from the Control (no light) to the LED conditions, for all spikes (black, $p=0.0046$, paired Wilcoxon signed-rank) and burst spikes (red, $\mathrm{p}=4.5 \mathrm{e}-4$, paired Wilcoxon signed-rank). Error bars represent mean +/- SEM. Right. Mean sensory-evoked Cortical multiunit (MU) firing rate from 102 trials (across 2 recordings, 1 mouse, $5 \mathrm{~ms}$ bin size). F. Example session of GEVI imaging following the delivery of a punctate whisker stimulus at time $t=0$, for the Control (no light, top row) and LED (bottom row) conditions. Images are averaged across 51 trials. Black bar represents $1 \mathrm{~mm}$. G. Left - Mean normalized peak GEVI sensory-evoked response was larger for the LED as compared to the Control condition ( $n=9$ mice, 13 recording sessions). For this plot, animals and sessions were each normalized to their control levels, and the LED condition is reported relative to the control. Left Inset - raw relative peak evoked \%dF/Fo, $p=0.017$, paired Wilcoxon signed-rank, $n=13$ recordings across 9 mice. Right Time series of the normalized sensory-evoked GEVI signal, generated from the integrated fluorescence within the $0.2 \times 0.2 \mathrm{~mm}$ ROI in the Control (gray) and LED (orange) conditions. Error bars represent mean $+/$ - SEM.

Figure 2. Thalamic baseline firing rate is invariant to optogenetic hyperpolarization via a tonic/burst switch. A. Experimental setup. Mice were injected with the viral vector eNphR3.0 (AAV5-CamllKianse-eNphR3.0-mCherry) targeting the ventral posterior-medial (VPm) region of the thalamus. On each trial, thalamic units were presented with light for $1.5 \mathrm{~s}$ (590 nm, LED approx. $17 \mathrm{~mW} / \mathrm{mm}^{2}$ ramp, Double LED approx. $35 \mathrm{~mW} / \mathrm{mm}^{2} \mathrm{ramp}$ - see Methods) from a $200 \mu \mathrm{m}$ optic fiber and recorded simultaneously with a single tungsten electrode. B. Example 
Borden et al., Thalamic state controls timing and synchronization of S1 cortex, 2021

1419 extracellular single trial rasters depicting effects of thalamic hyperpolarization on spiking in VPm 1420 thalamus. Black indicates tonic spikes; red indicates burst spikes. Recorded spike waveform 1421 shown at top. C. Top - Aggregate single-unit (SU, $n=5$ units) PSTH for all spikes (black) and burst 1422 spikes (red). Apparent is an initial decrease in overall firing rate following presentation of light, 1423 followed by a return to the pre-hyperpolarization level. Bottom - Aggregate multi-unit (MU) 1424 activity ( $n=9$ recordings). Bands represent are mean $+/$ - SEM. D. Left - Mean multi-unit thalamic firing rate over the $250-750 \mathrm{~ms}$ time window from the Control (no light) to the LED condition, for all MU spikes (black, Control vs LED $p=0.098$, Control vs Double LED $p=0.65$, paired Wilcoxon signrank test, $\mathrm{n}=9$ recordings). Middle - Mean single-unit thalamic firing rate over the $250-750 \mathrm{~ms}$ time window from the Control (no light, $n=8$ units) to the LED condition ( $n=5$ units), for all spikes (black, Control vs LED $p=0.55$, Control vs Double LED $p=0.37$, unpaired t-test) and burst spikes (red, Control vs LED $p=0.014$, Control vs Double LED $p=0.032$, unpaired t-test). Right - Increase in burst ratio from the Control to LED condition, where burst ratio is defined as the number of burst spikes divided by the total number of spikes (Control vs LED $p=0.0016$, Control vs Double LED $p=0.0016$, unpaired Wilcoxon rank sum test). E. Left - Example bursts for the Control and LED conditions. Right - Increase in the mean number of spikes per burst from the Control to the LED condition (Control vs LED $p=0.048$, Control vs Double LED $p=0.016$, unpaired Wilcoxon rank sum test, $n=5$, only considering cells with bursts). Error bars represent mean +/- SEM. F. Simulated spontaneous activity from Integrate-and-fire-or-burst (IFB) model with hyperpolarizing input at time $t=0$, showing all spikes (black) and burst spikes (red). See Methods and Supplemental Figure S3.

Figure 3. Cortical S1 baseline firing rate is invariant to optogenetic hyperpolarization of thalamus after transient increase. A. Experimental setup. Mice were injected with the viral vector eNphR3.0 (AAV5-CamllKianse-eNphR3.0-mCherry) targeting the ventral posterior-medial $(\mathrm{VPm})$ region of the thalamus. Thalamic units were continuously presented with light $(590 \mathrm{~nm}$, LED approx. $17 \mathrm{~mW} / \mathrm{mm}^{2}$ ramp, Double LED approx. $35 \mathrm{~mW} / \mathrm{mm}^{2}$ ramp) from a $200 \mu \mathrm{m}$ optic fiber, while cortical single-units were recorded simultaneously with a laminar multi-electrode within an identified cortical column (barrel) - see Methods. B. Top - Example extracellular rasters of cortical activity, depicting effects of thalamic hyperpolarization on cortical spiking, across trials and simultaneously recorded units (10 trials each from 15 units). Bottom - Aggregate PSTH across all recorded trials and cortical single-units $(n=118)$. Bands represent mean $+/-$ SEM. Highlighted are the transient and steady-state portions of the cortical response to the light. C. Mean cortical firing rate in the Control and LED conditions, showing an increase in the transient (open symbol, $300-350 \mathrm{~ms}$, Control vs LED $p=5.4 \mathrm{e}-14$, Control vs Double LED $p=1.4 \mathrm{e}-15$, Wilcoxon signed-rank test) and a return to near steady-state (closed symbol, 700-750ms, Control vs LED $p=0.2$, Control vs Double LED $p=0.013$, Wilcoxon signed-rank test) portions of the response. Error bars represent mean + /- SEM. eNphR3.0 (AAV5-CamlIKianse-eNphR3.0-mCherry) targeting the ventral posterior-medial (VPm) 
Borden et al., Thalamic state controls timing and synchronization of S1 cortex, 2021

1463 thalamic units were presented with light for $1.5 \mathrm{~s}\left(590 \mathrm{~nm}\right.$, LED approx. $17 \mathrm{~mW} / \mathrm{mm}^{2} \mathrm{ramp}$, 1464 Double LED approx. $35 \mathrm{~mW} / \mathrm{mm}^{2}$ ramp - see Methods) from a $200 \mu \mathrm{m}$ optic fiber, with LED 1465 illumination beginning at $0.75 \mathrm{~s}$ preceding stimulus delivery, and continuing for $0.75 \mathrm{~s}$ after 1466 stimulus delivery, while recording simultaneously with a single tungsten electrode. For cortical 1467 GEVI imaging, the entire cortical area was illuminated through the thinned skull at $465 \mathrm{~nm}$ with 1468 an LED and imaged with a CCD imaging setup (see Methods). B. Grand Multiunit PSTH for thalamic spiking in response to a single, punctate whisker stimulus $(t=0)$ for the Control (no light, top panel, $n=9$ recordings) and LED (bottom panel, $n=9$ recordings) conditions. Instantaneous firing rates (bin size $2 \mathrm{~ms}$ ) shown for all spikes (black). C. Mean sensory-evoked thalamic response over $0-30 \mathrm{~ms}$ time window for multi-unit recordings (Control vs LED $p=0.0039$, Control vs Double LED $p=0.0039$, unpaired Wilcoxon rank sum, $n=9$ ). Error bars represent mean $+/-$ SEM. D. Grand single unit PSTH for thalamic spiking in response to a single, punctate whisker stimulus $(t=0)$ for the Control (no light, top panel, $n=8$ units) and LED (bottom panel, $n=5$ units) conditions. Instantaneous firing rates (bin size $2 \mathrm{~ms}$ ) shown for all spikes (black) and putative burst spikes (red). E. Mean single-unit sensory-evoked thalamic burst ratio over the 0-30 ms time window (Control vs LED $p=0.011$, Control vs Double LED $p=0.019$, unpaired Wilcoxon rank sum Control $n=8$ units, LED conditions $n=5$ units). F. Example session of GEVI imaging following the delivery of a punctate whisker stimulus at time $t=0$, for the Control (no light, top row) and LED (bottom row) conditions. Images are averaged across 51 trials. G. Left - Peak GEVI sensory-evoked response (between $0-110 \mathrm{~ms}$ post stimulus) slightly decreased from the Control to the LED condition (Control vs LED $p=0.020$, Control vs Double LED $p=0.0039$, paired Wilcoxon signrank, $n=9$ recordings from 2 mice). Right - Mean normalized peak GEVI sensory-evoked response for the Control and LED conditions ( $n=9$ recordings). Before combining, animals and sessions were each normalized to their Control levels, and the LED condition is reported relative to the Control. H. In separate experiments, we hyperpolarized the thalamus while simultaneously recording the S1 Cortical response using a multichannel electrode. I. Cortical Grand PSTH evoked sensory response $(\mathrm{t}=0)$ across all recorded single units ( $\mathrm{n}=118$ units, $5 \mathrm{~ms}$ bins) for Control (black) and LED (orange) conditions. J. Left Average absolute evoked cortical response remained approximately invariant during LED on conditions across all cortical recorded units $(n=118)$. Evoked sensory response period defined as between $5-100 \mathrm{~ms}$ post stimulus. Right. Relative evoked cortical response decreased with increasing LED intensities (Control vs LED $p=0.20$, Control vs Double LED $p=0.034$, paired Wilcoxon signrank, $n=118$ units). Relative evoked response defined as the absolute response minus the preceding baseline activity $-50-0 \mathrm{~ms}$ pre-stimulus. Error bars represent mean +/- SEM.

Figure 5. Thalamic hyperpolarization sharpens the spatial and temporal cortical sensory evoked response revealed through GEVI imaging. A. GEVI imaging frames at peak response for the control (top) and LED (bottom) conditions for an example session. B. Cartoon illustration of the coupling between amplitude and spatial area in cortical imaging. Top - A reduction in evoked fluorescence amplitude can be qualitatively perceived as a reduction in spatial area of activation, due to the "iceberg" effect. Bottom - However, the spatial area of activation above a fixed threshold after normalization to the peak reveals the true effects on the area. C. Normalized 
Borden et al., Thalamic state controls timing and synchronization of S1 cortex, 2021

1507 Summary analyses (Error bars represent mean +/- SEM). Note, for this figure all conditions are 1508 normalized to their respective peak responses, as portrayed in B. Left - Mean spatial activation 1509 area of the normalized sensory-evoked response decreased from the Control to the LED condition 1510 (Control vs LED $p=0.025$, Control vs Double LED $p=0.046$, paired t-test, $n=9$ recordings). Middle 1511 The peak-to-baseline (defined in Figure 5C), calculated as the mean time from the peak of the 1512 fluorescence to the first return to pre-stimulus baseline (ms), decreased from the Control to the 1513 LED condition (Control vs LED $p=0.0059$, Control vs Double LED $p=0.0019$, unpaired t-test, $n=8$ 1514 Control recordings, 9 LED recordings, note one of the Control recordings did not return to 1515 baseline). Right - The recovery (defined in Figure 5C), calculated as the relative mean 1516 fluorescence during the time duration following the peak stimulus-evoked response (120$1517400 \mathrm{~ms}$ ), decreased from the Control to the LED condition (Control vs LED $p=0.016$, Control vs 1518 Double LED $p=0.020$, paired t-test, $\mathrm{n}=9$ recordings). LED approx. $17 \mathrm{~mW} / \mathrm{mm}^{2} \mathrm{ramp}$, Double LED approx. $35 \mathrm{~mW} / \mathrm{mm}^{2}$ ramp - see Methods. Error bars represent mean +/- SEM. responses in cortex. A. Cortical single-units were classified as Regular Spiking Units (RSUs, red) or Fast Spiking Units (FSUs, blue) based on the time interval from the peak-to-trough (see Methods). Example RSU and FSU waveforms are shown at the top, where bands represent $+/-1$ SD. The distribution of time intervals from peak-to-trough for the spike waveforms is shown at the bottom. B. PSTHs of the aggregate putative FSUs (left, $n=32$ ) and RSUs (right, $n=86$ ) in response to a punctate whisker deflection at time $\mathrm{t}=0$ (bin size $2 \mathrm{~ms}$ ) for the Control (black) and double LED (orange) conditions. C. Summary analyses (Error bars represent mean +/- SEM) for RSUs (top row) and FSUs (bottom row). Left - Mean baseline (-50 to Oms) RSU (Control vs LED $p=5.3 e-4$, Control vs Double LED $p=0.012$, Wilcoxon signed-rank test, $n=86$ ) and FSU (Control vs LED $p=0.15$, Control vs Double LED $p=0.30$, Wilcoxon signed-rank test, $n=32$ ) firing rates for the LED relative to the Control condition. Middle - RSU (Control vs LED $p=0.067$, Control vs Double LED $p=0.0082$, Wilcoxon signed-rank test, $n=86$ ) and FSU (Control vs LED, $p=0.30$, Control vs Double LED $p=0.45$, Wilcoxon signed-rank test, $n=32$ ) early sensory response $(5-50 \mathrm{~ms})$ for the LED compared to the Control conditions. Right - Late sensory response (60-100ms) for the LED compared to the Control condition for the RSU (Control vs LED $p=0.017$, Control vs Double LED $p=0.014, n=86$, Wilcoxon signed-rank test) and FSU (Control vs LED $p=2.2 e-4$, Control vs Double LED $p=4.5 e-4, n=32$, Wilcoxon signed-rank test) populations. LED approx. $17 \mathrm{~mW} / \mathrm{mm}^{2} \mathrm{ramp}$, Double LED approx. $35 \mathrm{~mW} / \mathrm{mm}^{2}$ ramp - see Methods. Error bars represent mean +/- SEM.

Figure 7. Thalamic hyperpolarization enhances cortical synchrony. A. The spike crosscorrelogram (CCG) was calculated as a histogram of spike times of cell 2 relative to a target spike of cell 1, repeated for all spikes of cell 1 . The pair-wise synchrony was calculated from the CCG as the area of the CCG between +/- 7.5ms, normalized by the total area of the CCG. B. Top Example Raster of two simultaneously collected FSU units, Cell 1 (black), Cell 2 (purple) for Control and LED conditions. Bottom. Synchronous spiking events only for the same neural pair (red, firing within $7.5 \mathrm{~ms}$ of each other). C. Aggregate cross-correlograms for FSUs ( $n=99$ pairs, see Methods) for the control (black) and LED (orange) conditions. Note that for the LED condition, the light level was $35 \mathrm{~mW} / \mathrm{mm}^{2}$ to better emphasize the change in synchrony with thalamic hyperpolarization. Cross-correlograms have been smoothed via a moving average filter, 2.5ms window. D. Mean 
Borden et al., Thalamic state controls timing and synchronization of S1 cortex, 2021

1551 levels of synchrony FSU pairs, for the spontaneous/baseline cortical firing as compared to the 1552 sensory-evoked response for the Control and LED conditions. Relative to the Control condition, 1553 thalamic hyperpolarization (LED, $35 \mathrm{~mW} / \mathrm{mm}^{\wedge} 2$ ) resulted in an increase in synchrony for the FSU 1554 (Control vs LED, $p=1.5 e-8$, Control vs Double LED, $p=3.5 e-13$, Wilcoxon signed-rank test, $n=99$ 1555 pairs) populations. LED approx. $17 \mathrm{~mW} / \mathrm{mm}^{2}$ ramp, Double LED approx. $35 \mathrm{~mW} / \mathrm{mm}^{2} \mathrm{ramp}-$ see 1556 Methods. Error bars represent mean +/- SEM.

Figure 8. A thalamocortical network model with TC synaptic adaptation reproduces three key experimental results. A. Thalamocortical model schematic (see Methods). A clustered network of excitatory (red) and inhibitory (blue) neurons received inputs from a model VPm barreloid, as well as random excitatory inputs. Thalamocortical synapses instantly depressed and slowly recovered in response to a VPm spike. B. Grand mean PSTHs for VPm tonic and burst spikes (top) and network excitatory and inhibitory neurons (bottom), for the Control (black) and LED (orange) conditions. Insets: same, but for 60-100 ms post-stimulus window. C. Left - Grand mean (+/- SEM) rates for all model VPm (black), excitatory (red) and inhibitory (blue) neurons for the early (0 - 50 ms post-stim, left) response window. VPm control: $28.08+/-2.04 \mathrm{~Hz}$; VPm LED: $34.53+/-2.67 \mathrm{~Hz} ; 23.0 \%$ increase, $\mathrm{p}=2.16 \times 10^{-7}$, Wilcoxon signed-rank test. Excitatory control: $11.24+/-0.3 \mathrm{~Hz}$; excitatory LED: $11.41+/-0.32 \mathrm{~Hz} ; 1.53 \%$ increase, $\mathrm{p}=0.34$. Inhibitory control: 143.39 +/- $2.85 \mathrm{~Hz}$; inhibitory LED: 142.73 +/- $2.76 \mathrm{~Hz}$; 0.46\% decrease, $p=0.61$. Right - Same, but for late (60-100 ms post-stim, right) response window. VPm control: $7.02+/-0.64 \mathrm{~Hz}$; VPm LED: $2.28+/-0.29 \mathrm{~Hz} ; 67.6 \%$ decrease, $\mathrm{p}=1.50 \times 10^{-7}$. Excitatory control: $3.23+/-0.11 \mathrm{~Hz}$; excitatory LED: $1.42+/-0.08 \mathrm{~Hz} ; 56.0 \%$ decrease, $\mathrm{p}=4.94 \times 10^{-90}$. Inhibitory control: $47.38+/-$ $1.71 \mathrm{~Hz}$; inhibitory LED: $17.95+/-1.27 \mathrm{~Hz}$; $62.1 \%$ decrease, $\mathrm{p}=3.86 \times 10^{-18}$. D. Grand crosscorrelograms for 147 randomly-selected, valid pairs of network excitatory neurons (see Model Supplement Section 1). E. Left - Same as in (D), but for window used to calculate synchrony (+/$7.5 \mathrm{~ms}$ ). Right - Grand mean pairwise synchrony for excitatory pairs used to generate CCGs in $D$, left. Exc-exc synchrony control: 0.56 +/- 0.02; exc-exc synchrony LED: $0.66+/-0.02 ; p=3.03 \times 10^{-}$ ${ }^{7}$, control vs. LED. F, G. Same as in (D, E), but for 200 randomly-selected, valid network inhibitory neurons. Inh-inh synchrony control: $0.53+/-0.003$; inh-inh synchrony LED: $0.60+/-0.003 ; \mathrm{p}=$ $1.44 \times 10^{-34}$, control vs. LED. * Indicates $0.01 \leq p<0.05, * *$ indicates $0.001 \leq p<0.01, * * *$ indicates $p<0.001$, Wilcoxon signed-rank test. 


\section{Figure 1}

A

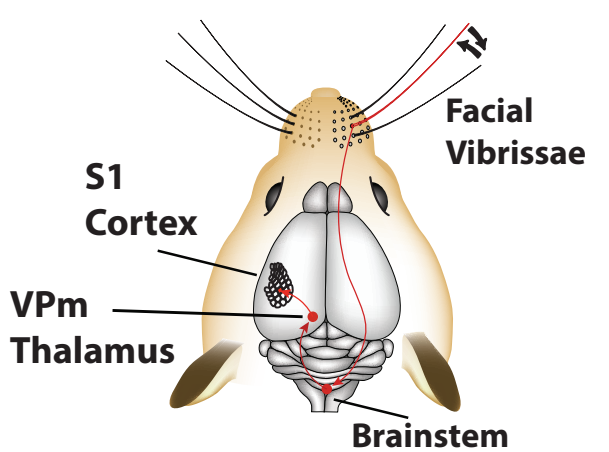

B

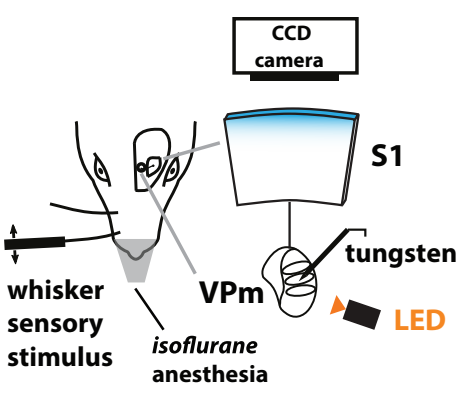

E anesth. VPm grand PSTH

D

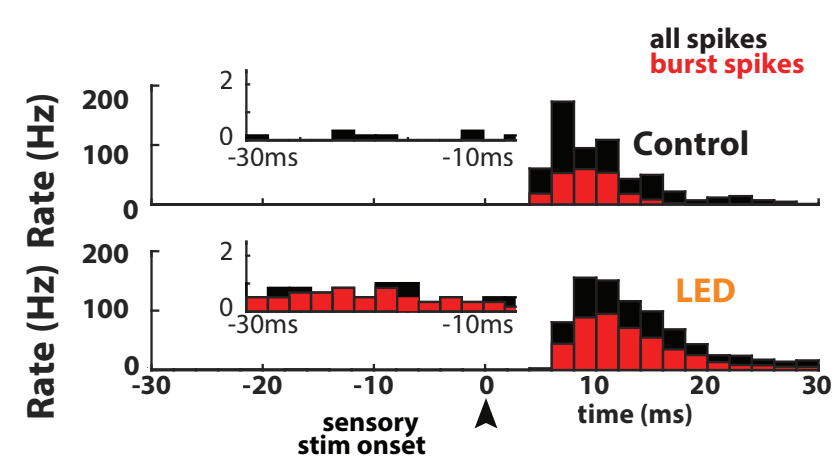

$\mathbf{F}$

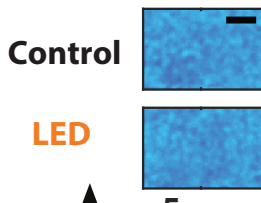

sensory stim onset anesth. CTX evoked GEVI response

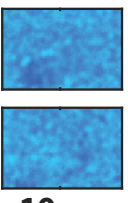

$10 \mathrm{~ms}$

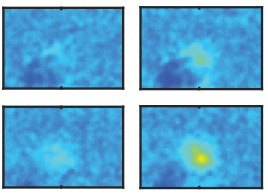

$15 \mathrm{~ms}$
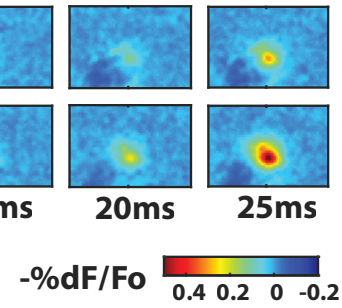

C

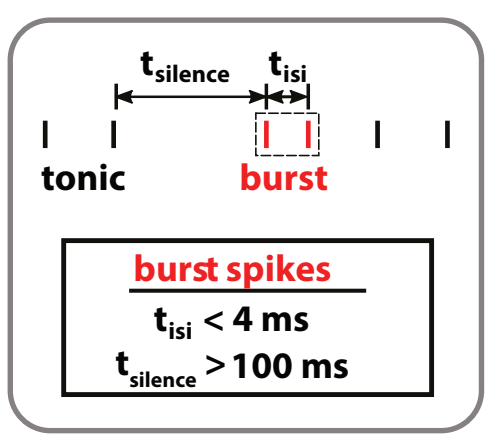

anesth. cortical MU recordings

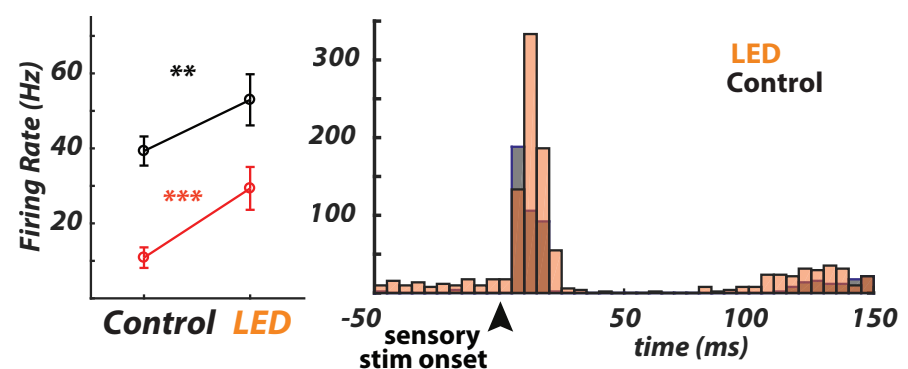

anesth. CTX evoked GEVI response
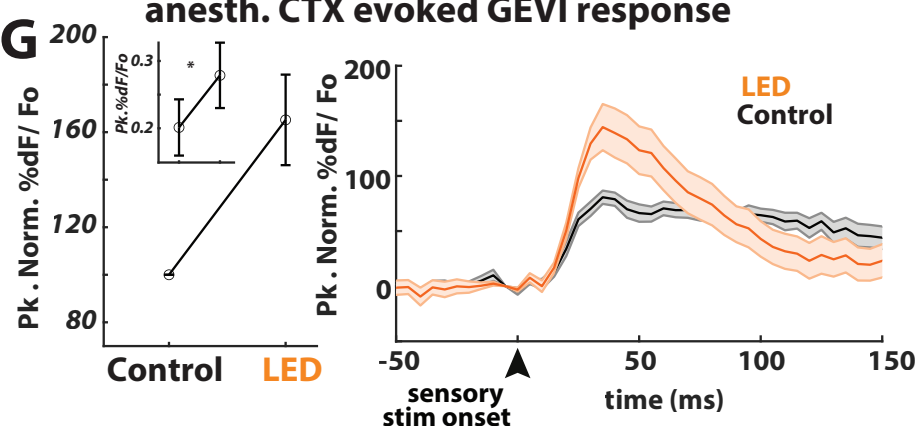


\section{Figure 2}

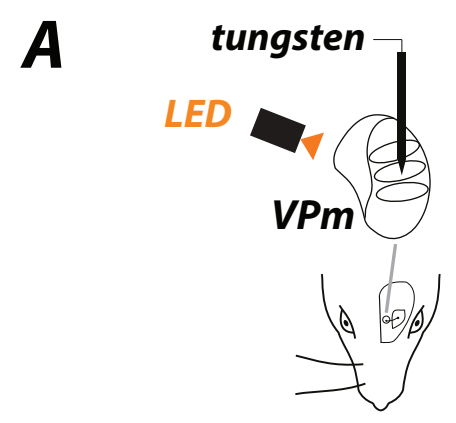

B
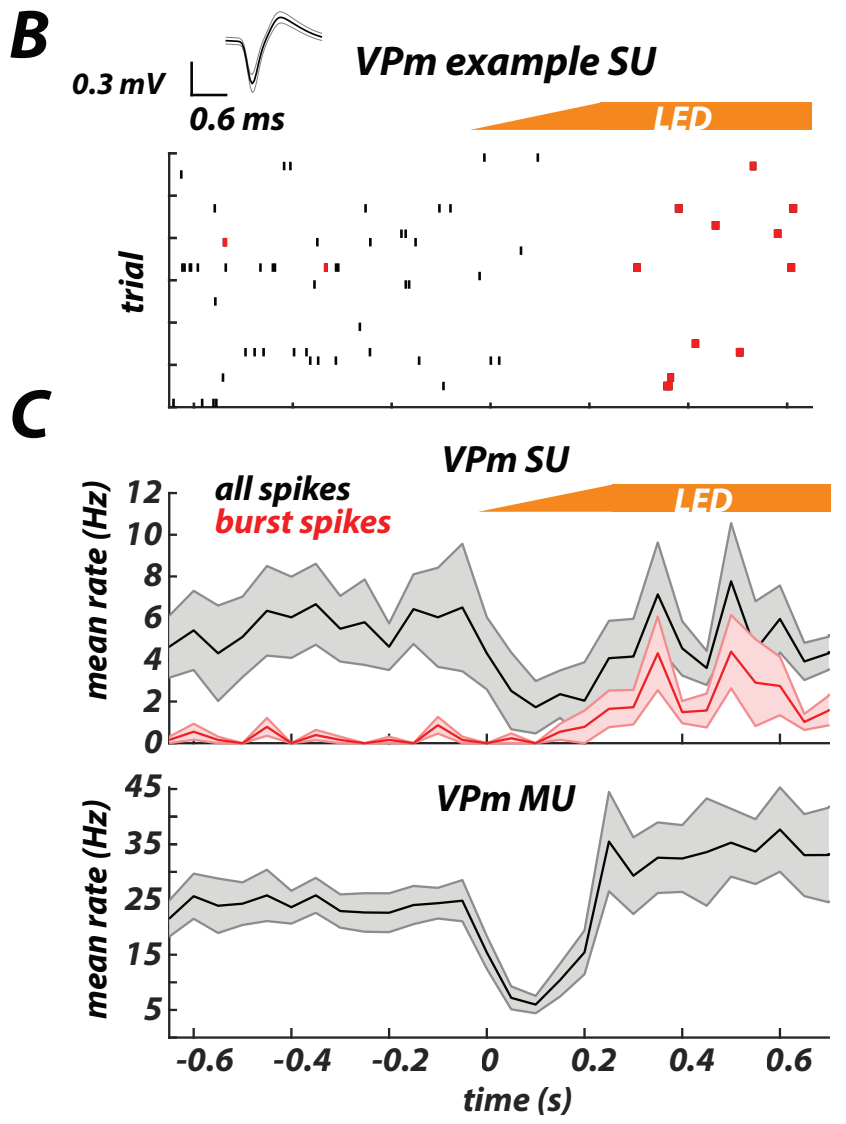

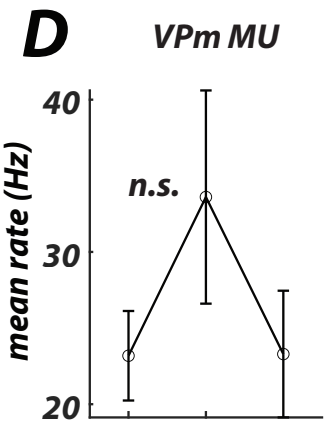

Control LED YLLED
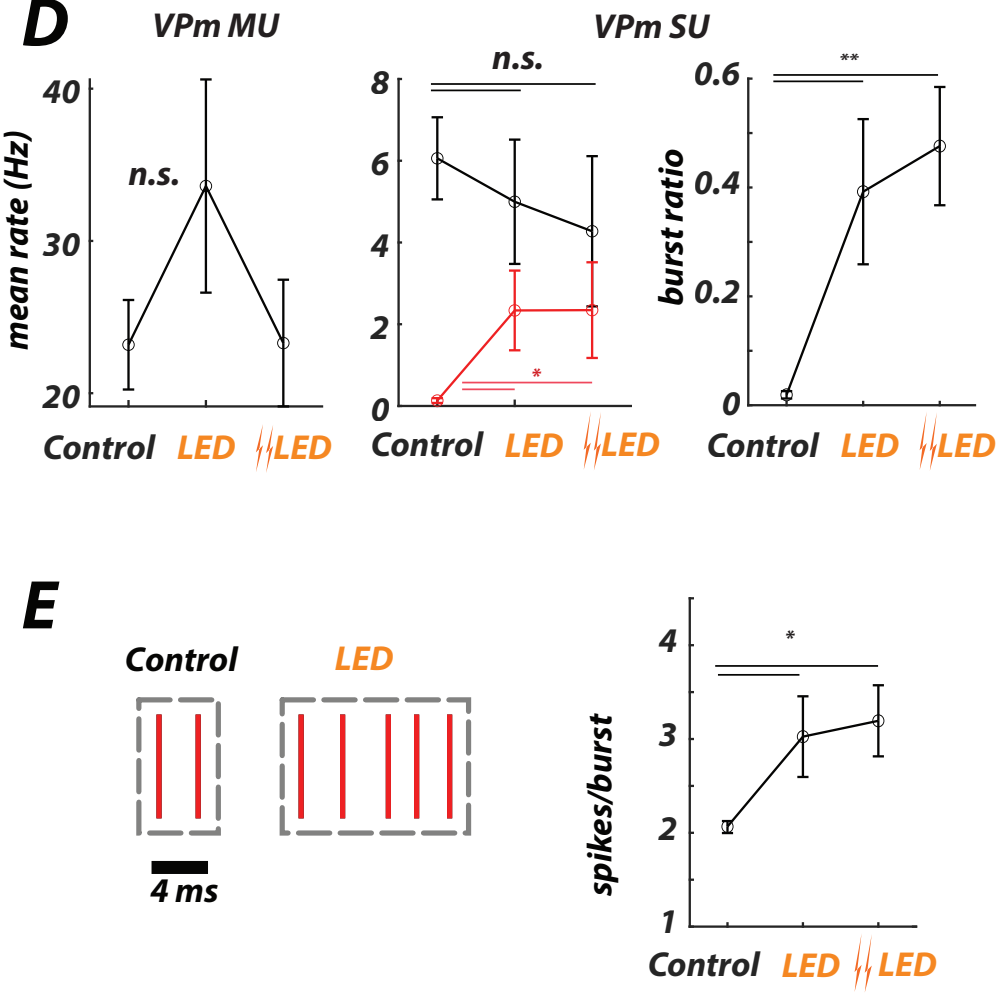

$F$

IFB model

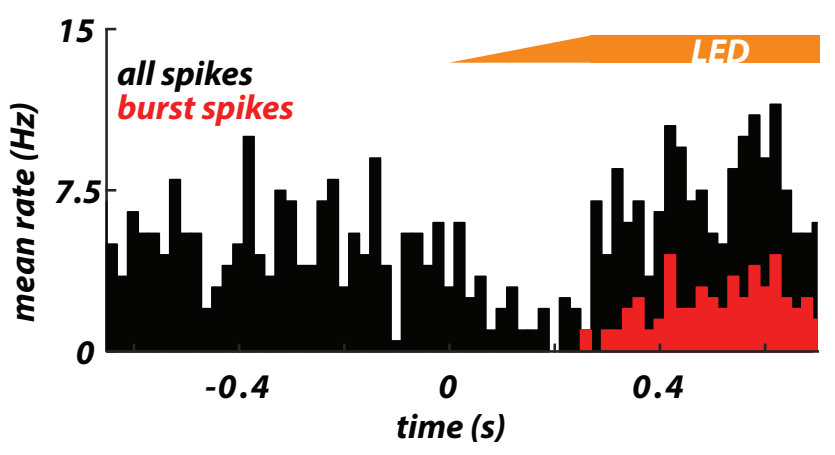




\section{Figure 3}

A

A silicon

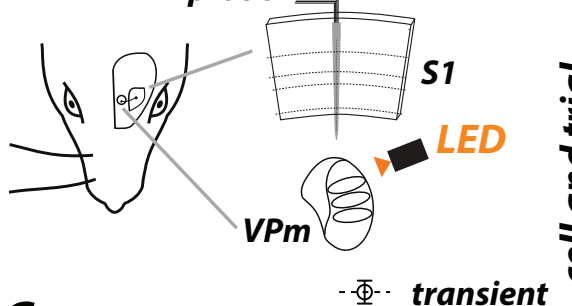

C

B S1 example session
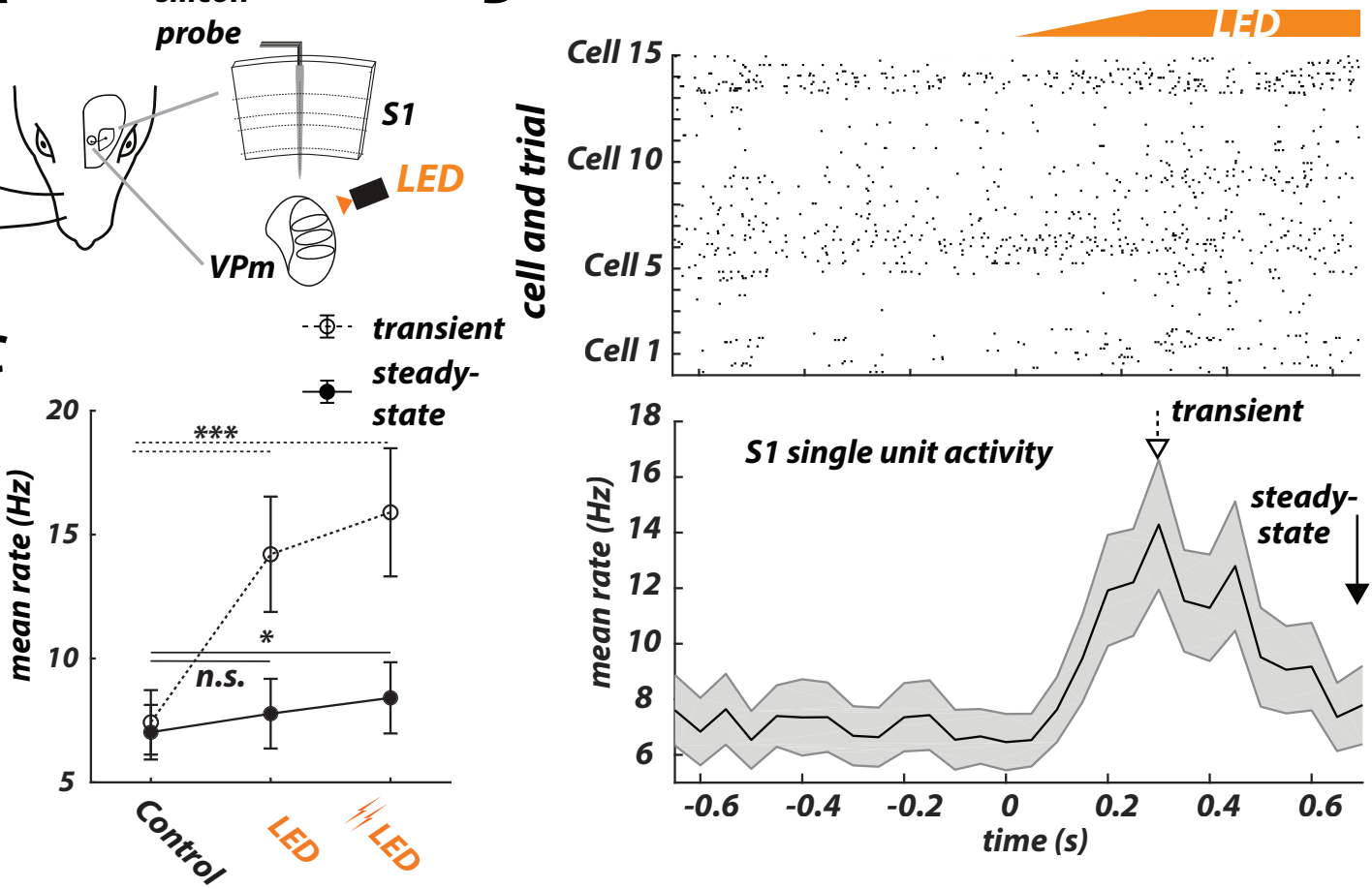


\section{Figure 4}
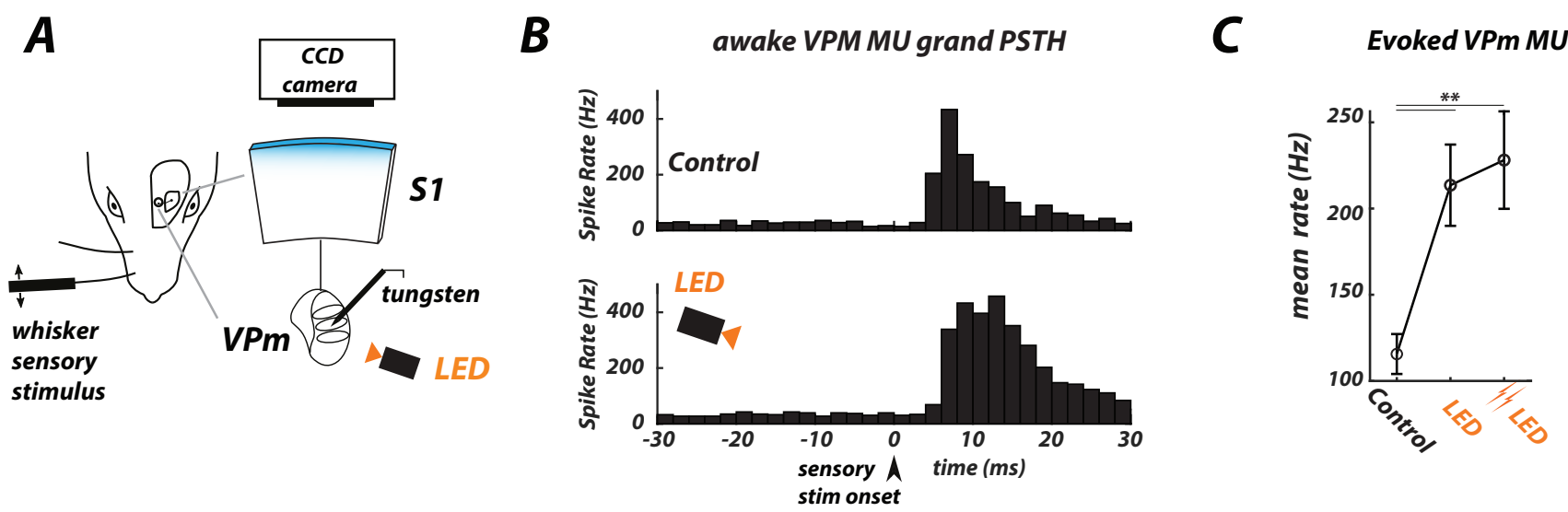

D
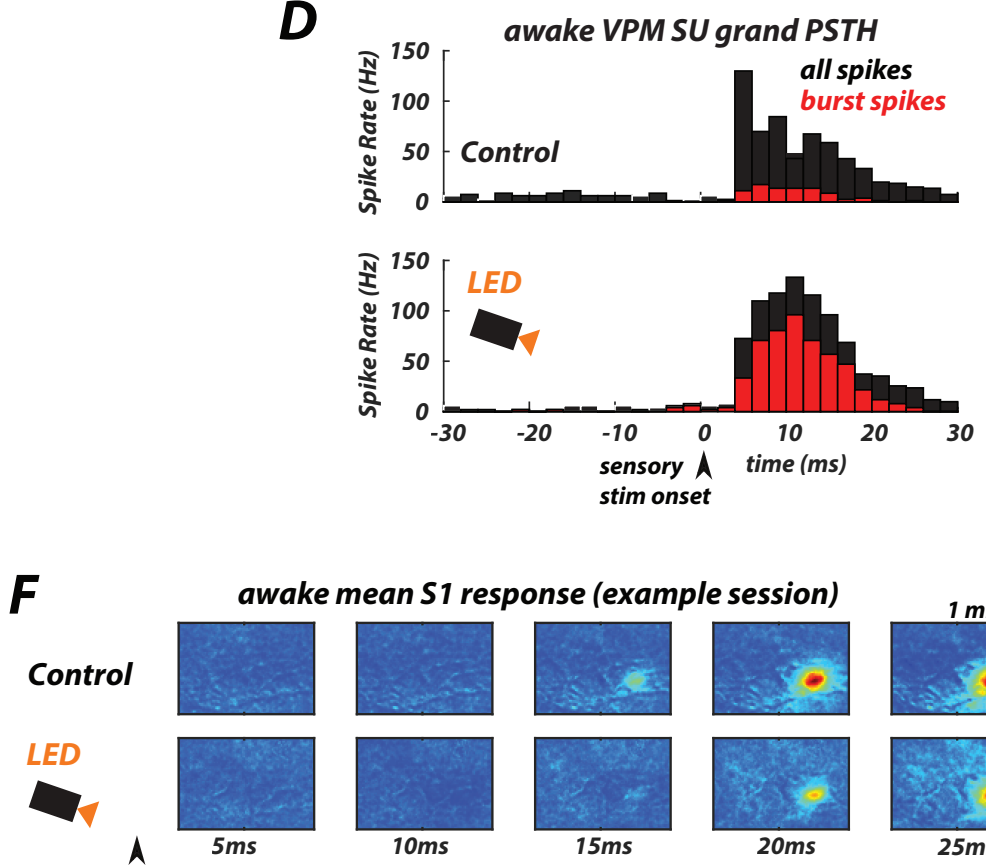

sensory stim

onset

\section{H}

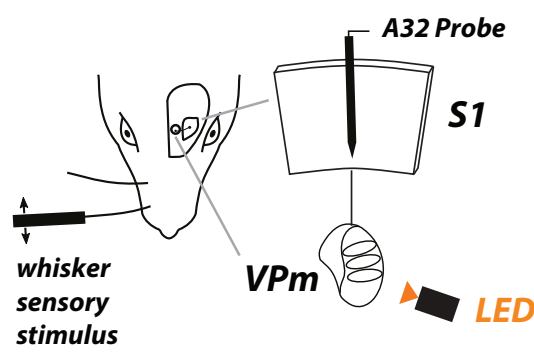

E

Evoked VPm SU
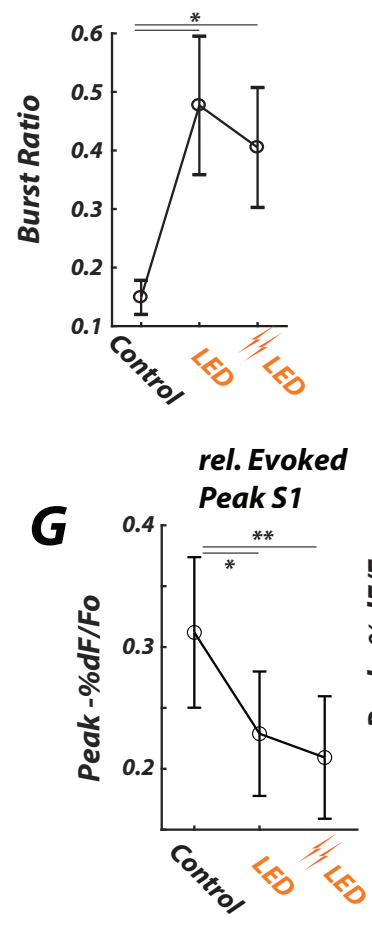

rel. Evoked

Norm. Peak S1

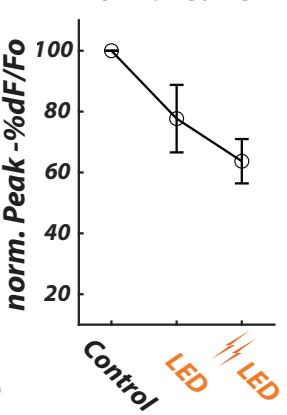

rel. Evoked

abs. Evoked (5-100ms)

(Response-Baseline)
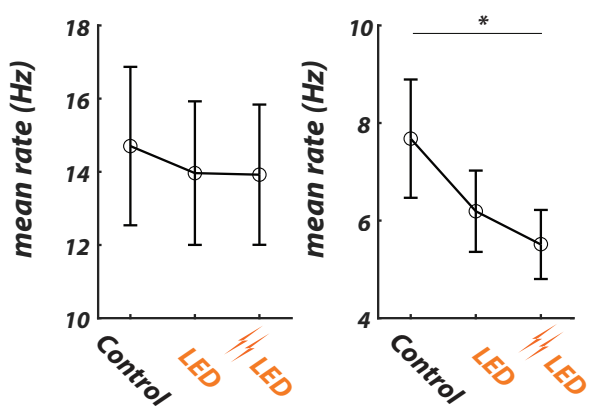
bioRxiv preprint doi: https://doi.org/10.1101/2021.07.09.451656; this version posted July 10, 2021. The copyright holder for this preprint (which was not certified by peer review) is the author/funder, who has granted bioRxiv a license to display the preprint in perpetuity. It is made

\section{Figure 5}

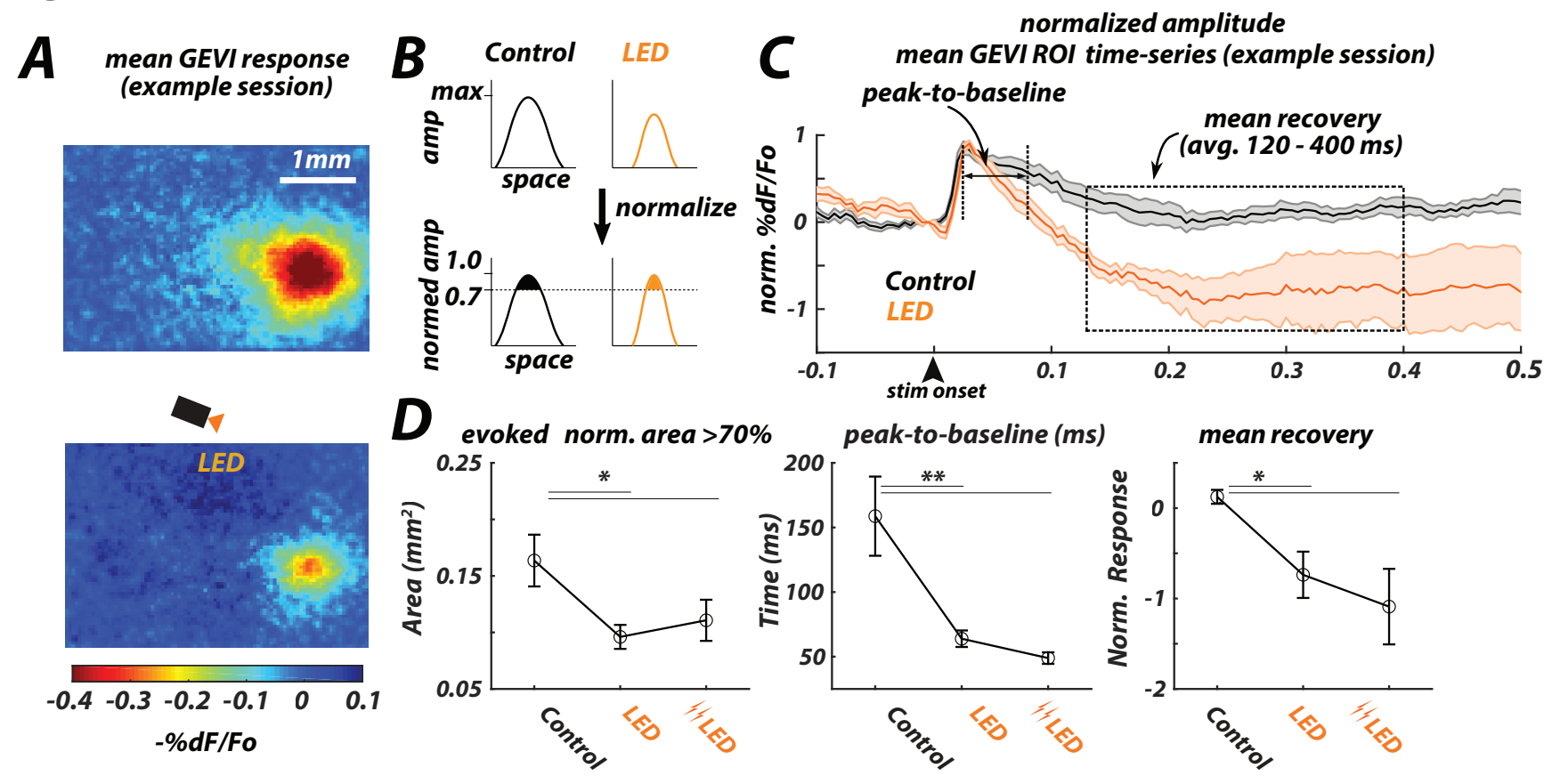


Figure 6

A
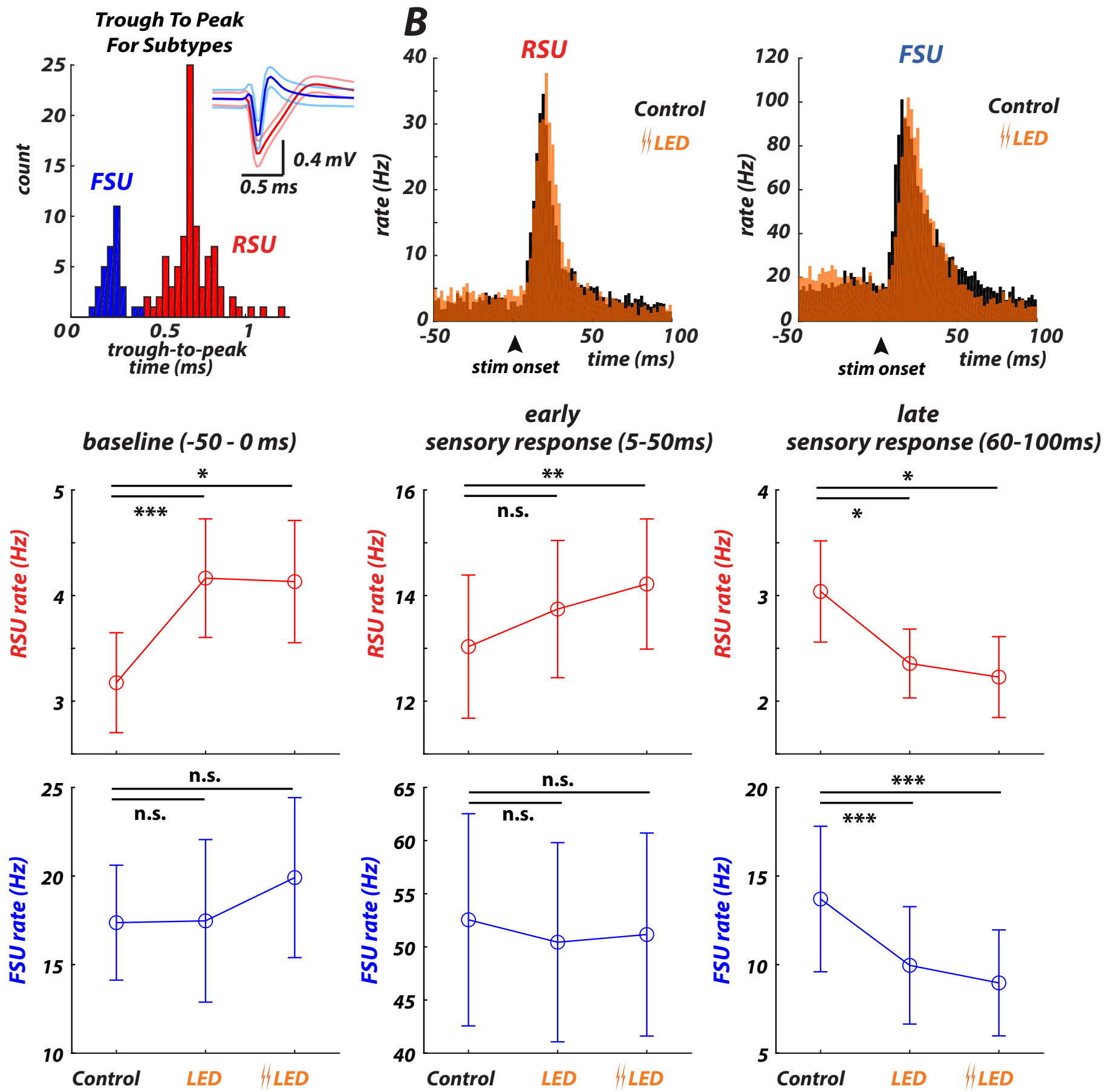
bioRxiv preprint doi: https://doi.org/10.1101/2021.07.09.451656; this version posted July 10, 2021. The copyright holder for this preprint (which

was not certified by peer review) is the author/funder, who has granted bioRxiv a license to display the preprint in perpetuity. It is made available under aCC-BY-NC 4.0 International license.

\section{Figure 7}

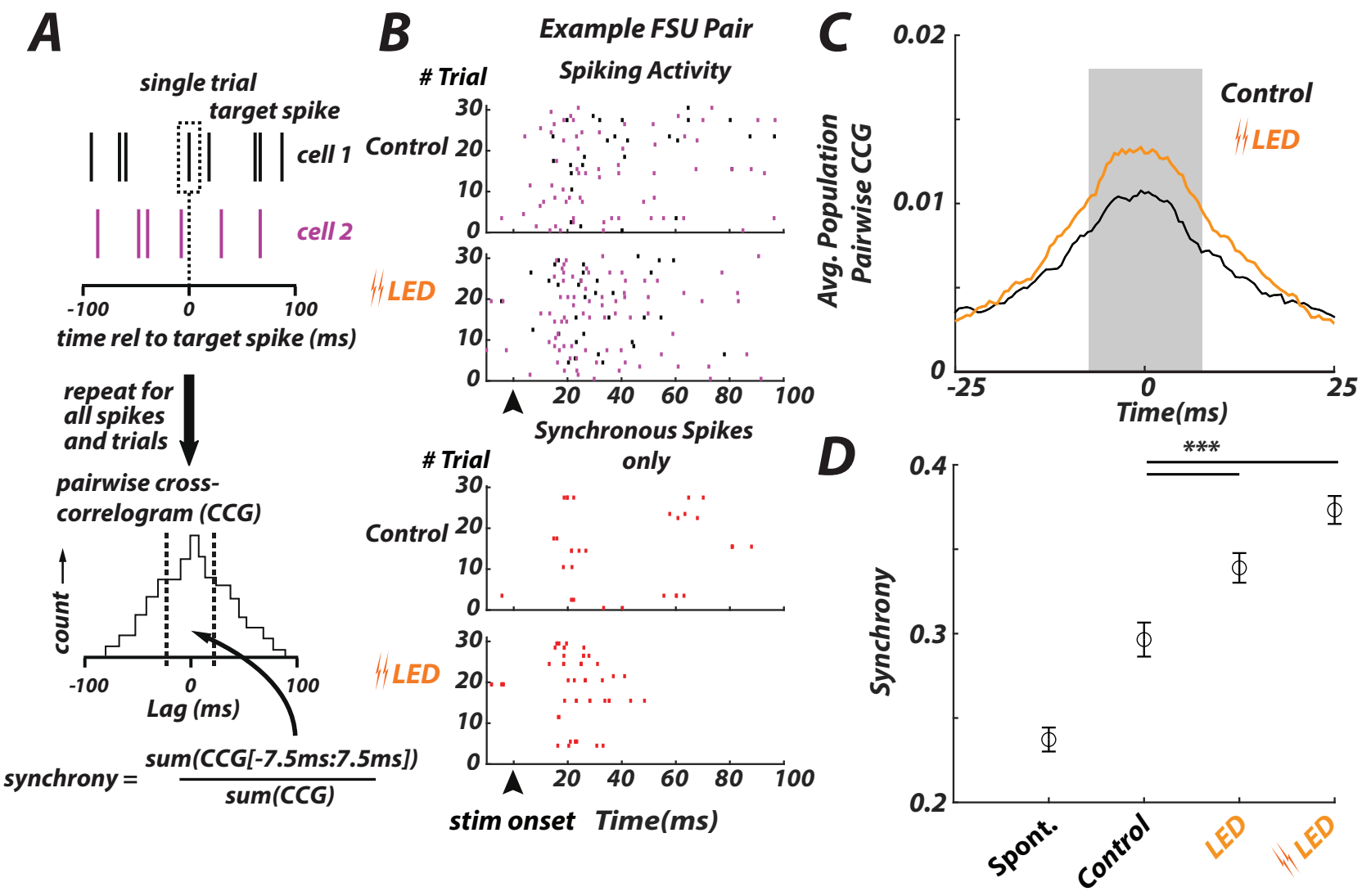


Figure 8
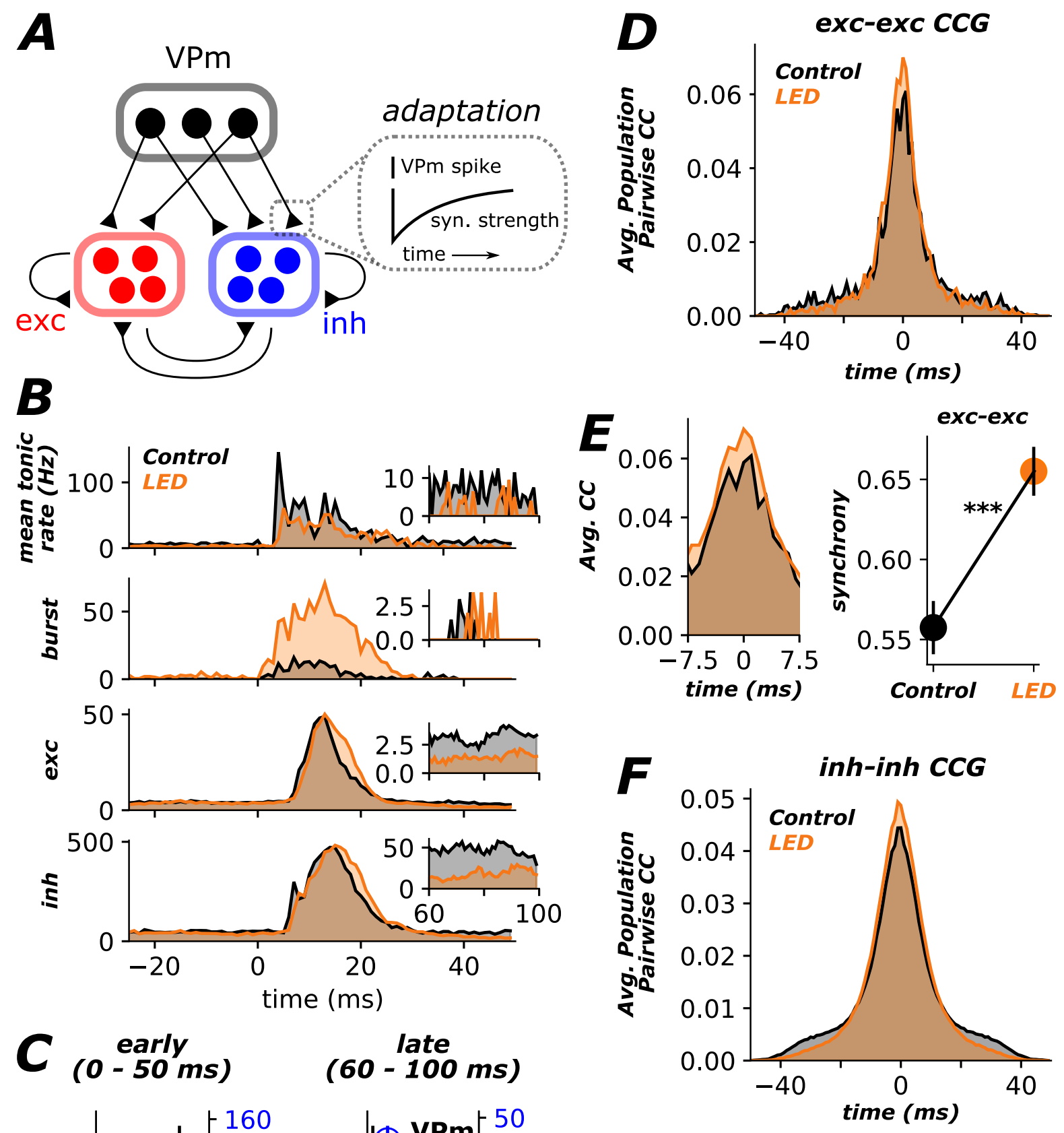

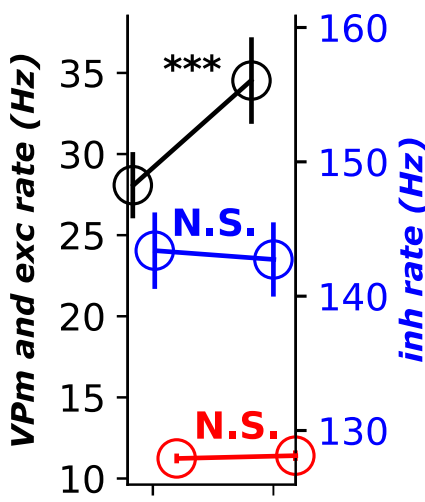

Control $L E D$
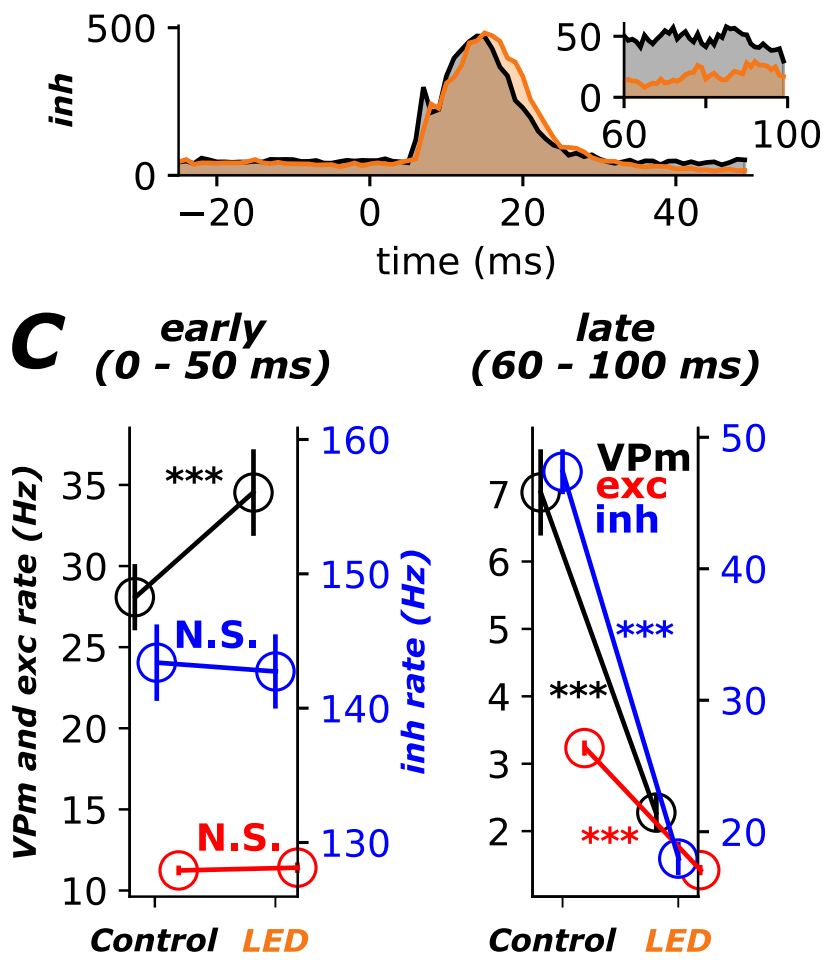
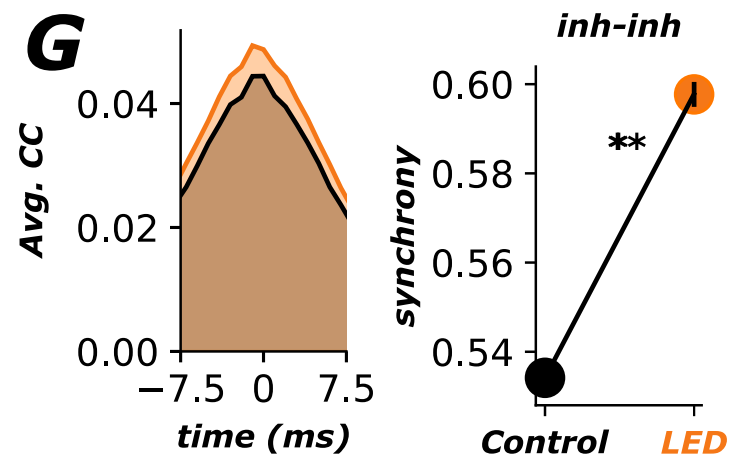